\title{
Adult dentate gyrus neurogenesis: a functional model
}

\author{
Olivia Gozel ${ }^{1,2, *}$ and Wulfram Gerstner ${ }^{1}$ \\ ${ }^{1}$ Laboratory of Computational Neuroscience, School of Life Sciences and School of \\ Computer and Communication Sciences, Brain-Mind Institute, Ecole Polytechnique \\ Fédérale de Lausanne, Lausanne, Switzerland \\ ${ }^{2}$ Current affiliation: Department of Neurobiology, University of Chicago, Chicago, IL \\ 60637, USA \\ *gozel@uchicago.edu
}

\section{Summary}

In adult dentate gyrus neurogenesis, the link between maturation of newborn neurons and their function, such as behavioral pattern separation, has remained puzzling. By analyzing a theoretical model, we show that the switch from excitation to inhibition of the GABAergic input onto maturing newborn cells is crucial for their proper functional integration. When the GABAergic input is excitatory, cooperativity drives the growth of synapses such that newborn cells become sensitive to stimuli similar to those that activate mature cells. When GABAergic input switches to inhibitory, competition pushes the configuration of synapses onto newborn cells towards stimuli that are different from previously stored ones. This enables the maturing newborn cells to code for concepts that are novel, yet similar to familiar ones. Our theory of newborn cell maturation explains both how adult-born dentate granule cells integrate into the preexisting network and why they promote separation of similar but not distinct patterns.

\section{Introduction}

In the adult mammalian brain, neurogenesis, the production of new neurons, is restricted to a few brain areas, such as the olfactory bulb and the dentate gyrus (Deng et al. 2010). The dentate gyrus is a major entry point of input from cortex, primarily entorhinal cortex (EC), to the hippocampus (Amaral et al., 
2007), which is believed to be a substrate of learning and memory (Jarrard, 1993). Adult-born cells in dentate gyrus mostly develop into dentate granule cells (DGCs), the main excitatory cells that project to area CA3 of hippocampus (Deng et al., 2010).

The properties of rodent adult-born DGCs change as a function of their maturation stage, until they become indistinguishable from other mature DGCs at approximately 8 weeks (Deng et al., 2010; Johnston et al., 2016) (Fig. 1a). Many of them die before they fully mature (Dayer et al., 2003). Their survival is experience-dependent, and relies upon NMDA receptor activation (Tashiro et al., 2006). Initially, newborn DGCs have enhanced excitability (Schmidt-Hieber et al. 2004 Li et al., 2017) and stronger synaptic plasticity than mature DGCs, reflected by a larger LTP amplitude and a lower threshold for induction of LTP (Wang et al., 2000, Schmidt-Hieber et al., 2004; Ge et al., 2007). Furthermore, after 4 weeks of maturation adult-born DGCs have only weak connections to interneurons, while at 7 weeks of age their activity causes indirect inhibition of mature DGCs (Temprana et al., 2015).

Newborn DGCs receive no direct connections from mature DGCs (Deshpande et al., 2013; Alvarez et al., 2016) (yet see (Vivar et al., 2012)), but are indirectly activated via interneurons (Alvarez et al., 2016; Heigele et al., 2016). At about three weeks after birth, the $\gamma$-aminobutyric acid (GABAergic) input from interneurons to adult-born DGCs switches from excitatory in the early phase to inhibitory in the late phase of maturation (Ge et al., 2006; Deng et al., 2010) ('GABA-switch', Fig. 1 1). Analogous to a similar transition during embryonic and early postnatal stages (Wang and Kriegstein, 2010), the GABA-switch is caused by a change in the expression profile of chloride cotransporters. In the early phase of maturation, newborn cells express the $\mathrm{Na}^{+}-\mathrm{K}^{+}-2 \mathrm{Cl}^{-}$cotransporter $\mathrm{NKCC} 1$, which leads to a high intracellular chloride concentration. Hence the GABA reversal potential is higher than the resting potential (Ge et al., 2006; Heigele et al., 2016), and GABAergic inputs lead to $\mathrm{Cl}^{-}$ions outflow through the $\mathrm{GABA}_{A}$ ionic receptors, which results in depolarization of the newborn cell (Ben-Ari, 2002; Owens and Kriegstein, 2002). In the late phase of maturation, expression of the $\mathrm{K}^{+}-\mathrm{Cl}^{-}$coupled cotransporter KCC2 kicks in, which lowers the intracellular chloride concentration of the newborn cell to levels similar to those of mature cells, leading to a hyperpolarization of the cell membrane due to $\mathrm{Cl}^{-}$inflow upon GABAergic stimulation (Ben-Ari, 2002; Owens and Kriegstein, 2002). The transition from depolarizing (excitatory) to hyperpolarizing (inhibitory) effects of GABA is referred to as the 'GABA-switch'. It has been shown that GABAergic inputs are crucial for the integration of newborn DGCs into the preexisting circuit (Ge et al., 2006; Chancey et al., 2013; Alvarez et al., 2016; Heigele et al., 2016).

The mammalian dentate gyrus contains - just like hippocampus in general a myriad of inhibitory cell types (Freund and Buzsáki, 1996; Somogyi and Klaus- 
berger, 2005; Klausberger and Somogyi, 2008) including basket cells, chandelier cells, and hilar cells. Basket cells can be subdivided in two categories: some express cholecystokinin (CCK) and vasoactive intestinal polypeptide (VIP), while the others express parvalbumin (PV) and are fast-spiking (Freund and Buzsáki, 1996: Amaral et al., 2007). Chandelier cells also express PV (Freund and Buzsáki, 1996). Overall, it has been estimated that PV is expressed in 15-21\% of all dentate GABAergic cells (Freund and Buzsáki, 1996), and in 20-25\% of the GABAergic neurons in the granule cell layer (Houser, 2007). Amongst the GABAergic hilar cells, $55 \%$ express somatostatin (SST) (Houser, 2007) [and somatostatin-positive interneurons (SST-INs) represent about $16 \%$ of the GABAergic neurons in the dentate gyrus as a whole (Freund and Buzsáki, 1996)]. While axons of hilar interneurons (HIL) (Yuan et al., 2017) stay in the hilus and provide perisomatic inhibition onto dentate GABAergic cells (Yuan et al., 2017), axons of hilar-perforantpath-associated interneurons (HIPP) extend to the molecular layer and provide dendritic inhibition onto both DGCs and interneurons (Yuan et al., 2017). HIPP axons generate lots of synaptic terminals and extend as far as $3.5 \mathrm{~mm}$ along the septotemporal axis of the dentate gyrus (Amaral et al. 2007). PV-expressing interneurons (PV-INs) and SST-INs both target adult-born DGCs early (after 2-3 weeks) in their maturation (Groisman et al., 2020). PV-INs provide both feedforward inhibition and feedback inhibition (also called lateral inhibition) to the DGCs (Groisman et al., 2020). In general, SST-INs provide lateral, but not feedforward, inhibition onto DGCs (Stefanelli et al., 2016; Groisman et al., 2020).

Adult-born DGCs are preferentially reactivated by stimuli similar to the ones they experienced during their early phase of maturation, up to 3 weeks after cell birth (Tashiro et al., 2007). Even though the amount of newly generated cells per month is rather low (3 to $6 \%$ of the total DGCs population (Van Praag et al., 1999; Cameron and McKay, 2001)), adult-born DGCs are critical for behavioral pattern separation (Clelland et al., 2009; Sahay et al., 2011a; Jessberger et al., 2009), in particular in tasks where similar stimuli or contexts have to be discriminated (Clelland et al., 2009; Sahay et al., 2011a). However, the functional role of adult-born DGCs is controversial (Sahay et al., 2011b; Aimone et al., 2011). One view is that newborn DGCs contribute to pattern separation through a modulatory role (Sahay et al., 2011b). Another view suggests that newborn DGCs act as encoding units that become sensitive to features of the environment which they encounter during a critical window of maturation (Kee et al., 2007; Tashiro et al., 2007). Some authors have even challenged the role of newborn DGCs in pattern separation in the classical sense and have proposed a pattern integration effect instead (Aimone et al., 2011). Within that broader controversy, we ask two specific questions: First, why are GABAergic inputs crucial for the integration of newborn DGCs into the preexisting circuit? And second, why are newborn DGC particularly important in tasks where similar stimuli or contexts have to be discriminated? 
To address these questions, we present a model of how newborn DGCs integrate into the preexisting circuit. In contrast to earlier models where synaptic input connections onto newborn cells were assumed to be strong enough to drive them (Chambers et al., 2004, Becker, 2005, Crick and Miranker, 2006; Wiskott et al., 2006: Chambers and Conroy, 2007; Aimone et al., 2009; Appleby and Wiskott, 2009; Weisz and Argibay, 2009, 2012; Temprana et al., 2015; Finnegan and Becker, 2015, DeCostanzo et al., 2019), our model uses an unsupervised biologically plausible Hebbian learning rule that makes synaptic connections either disappear or grow from small values at birth to values that eventually enable feedforward input from EC to drive DGCs. Contrary to previous modeling studies, our plasticity model does not require an artificial renormalization of synaptic connection weights since model weights are naturally bounded by homeostatic heterosynaptic plasticity. We show that learning with a biologically plausible plasticity rule is possible thanks to the GABA-switch, which has been overlooked in previous modeling studies. Specifically the growth of synaptic weights from small values is supported in our model by the excitatory action of GABA whereas, after the switch, specialization of newborn cells arises from competition between DGCs, triggered by the inhibitory action of GABA. Furthermore, our theory of adultborn DGCs integration yields a transparent explanation of why newborn cells favor pattern separation of similar stimuli, but do not impact pattern separation of distinct stimuli.

\section{Results}

We model a small patch of cells within dentate gyrus as a recurrent network of 100 DGCs and 25 GABAergic interneurons, omitting the Mossy cells for the sake of simplicity (Fig. 1b). The modeled interneurons correspond to SST-INs from the HIPP category, as they are the providers of feedback inhibition to DGCs through dendritic projections (Stefanelli et al., 2016; Yuan et al., 2017; Groisman et al., 2020). The activity of a DGC with index $i$ and an interneuron with index $k$ is described by their continuous firing rates $\nu_{i}$ and $\nu_{k}^{I}$, respectively. Connectivity in a localized patch of dentate neurons is high: DGCs densely project to GABAergic interneurons (Acsády et al., 1998), and SST-INs heavily project to cells in their neighborhood (Amaral et al., 2007). Hence, in the recurrent network model, each model DGC projects to, and receives input from, a given interneuron with probability 0.9. The exact percentage of GABAergic neurons (or SST-INs) in the dentate gyrus as a whole is not known, but has been estimated at about $10 \%$ and only a fraction of these are SST-INs (Freund and Buzsáki, 1996). The number of inhibitory neurons in our model network might therefore seem too high. However, our results are robust to substantial changes in the number of inhibitory neurons (Suppl. Table S2). 
Each of the 100 model DGCs receives input from a set of 144 model EC cells (Fig. 1b). In the rat the number of DGCs has been estimated to be about $10^{6}$, while the number of EC input cells is estimated to be about $2 \cdot 10^{5}(\mathrm{An}-$ dersen et al. 2007), yielding an expansion factor from EC to dentate gyrus of about 5 . Theoretical analysis suggests that the expansion of the number of neurons enhances decorrelation of the representation of input patterns (Marr, 1969; Albus, 1971; Marr, 1971; Rolls and Treves, 1998), and promotes pattern separation (Babadi and Sompolinsky, 2014). Our standard network model does not reflect this expansion, because we want to highlight the particular ability of adult neurogenesis in combination with the GABA-switch to decorrelate input patterns independently of specific choices of the network architecture. However, we show later that an enlarged network with an expansion from 144 model EC cells to 700 model DGCs (similar to the anatomical expansion factor) yields similar results.

At birth a DGC with index $i$ does not receive synaptic glutamatergic input yet. Hence the connection from any model EC cell with index $j$ is initialized at $w_{i j}=0$. The growth or decay of the synaptic strength $w_{i j}$ of the connection from $j$ to $i$ is controlled by a Hebbian plasticity rule (Fig. 1 c):

$$
\Delta w_{i j}=\eta\left\{x_{j} \cdot \operatorname{LTP}\left(\nu_{i}-\theta\right)-x_{j} \cdot \operatorname{LTD}\left(\theta-\nu_{i}\right)-w_{i j} \cdot \operatorname{HET}\left(\nu_{i}-\theta\right)\right\}
$$

where $x_{j}$ is the firing rate of the presynaptic EC neuron and $\eta$ ('learning rate') is the susceptibility of a cell to synaptic plasticity. The first term on the righthand-side of equation (1) describes Long-Term-Potentiation (LTP) whenever the presynaptic neuron is active $\left(x_{j}>0\right)$ and the postsynaptic firing $\nu_{i}$ is above a threshold $\theta$; the second term on the right-hand-side of equation (1) describes Long-Term-Depression (LTD) whenever the presynaptic neuron is active and the postsynaptic firing rate is positive but below the threshold $\theta$; LTD stops if the synaptic weight is zero. Such a combination of LTP and LTD is consistent with experimental data (Artola et al., 1990; Sjöström et al., 2001) as shown in earlier rate-based (Bienenstock et al., 1982) or spike-based (Pfister and Gerstner, 2006) plasticity models. The third term on the right-hand-side of equation (1) implements heterosynaptic (HET) plasticity (Chistiakova et al., 2014:Zenke and Gerstner, 2017) whenever the postsynaptic neuron fires at a rate above $\theta$, independent of presynaptic activity (Methods). It ensures that the weights cannot grow without bounds (Methods). Since survival of newborn cells requires NMDA receptor activation (Tashiro et al. 2006), a DGC which has not been able to grow several strong weights is removed after some time and replaced by another newborn DGC.

We ask whether such a biologically-plausible plasticity rule enables adult-born DGCs to be integrated in an existing network of mature cells. To address this question, we exploit two observations (Fig. 1 $)$ : first, the effect of interneurons onto newborn DGCs exhibits a GABA-switch from excitatory to inhibitory after about three weeks of maturation (Ge et al., 2006; Deng et al., 2010) and, second, 
newborn DGCs receive input from interneurons early in their maturation (before the third week), but project back to interneurons only later (Temprana et al. 2015). However, before integration of adult-born DGCs can be addressed, an adult-stage network where mature cells already store some memories has to be constructed.

\section{Mature neurons represent prototypical input patterns}

In an adult-stage network, some mature cells already have a functional role. Hence we pretrain our network of 100 DGCs using the same learning rule equation (1) that we will use later for the integration of newborn cells. For the stimulation of EC cells, we apply patterns representing thousands of handwritten digits in different writing styles from MNIST, a standard data set in artificial intelligence (LeCun et al., 1998). Even though we do not expect EC neurons to show a 2-dimensional arrangement, the use of 2-dimensional patterns provides a simple way to visualize the activity of all 144 EC neurons in our model (Fig. 1d $)$. We implicitely model feedforward inhibition from PV-INs (Groisman et al., 2020) by normalizing the L2-norm of each input pattern to unity (Methods). Below, we present results for a representative combination of three digits (digits 3, 4 and 5), but other combinations of digits have also been tested (Suppl. Table S1).

After pretraining with patterns from digits 3 and 4 in a variety of writing styles, we examine the receptive field of each DGC. Each receptive field, consisting of the connections from all 144 EC neurons onto one DGC, is characterized by its spatial structure (i.e., the pattern of connection weights) and its total strength (i.e., the efficiency of the optimal stimulus to drive the cell). We observe that out of the 100 DGCs, some have developed spatial receptive fields that correspond to different writing styles of digit 3, others receptive fields that correspond to variants of digit 4 (Fig. 1e).

Behavioral discrimination has been shown to be correlated with classification accuracy based on DGC population activity (Woods et al., 2020). Hence, to quantify the representation quality, we compute classification performance by a linear classifier that is driven by the activity of our 100 DGC model cells (Methods). At the end of pretraining, the classification performance for patterns of digits 3 and 4 from a distinct test set not used during pretraining is high: $99.25 \%$ (classification performance on digit 3: 98.71\%; digit 4: 99.80\%), indicating that nearly all input patterns of the two digits are well represented by the network of mature DGCs. The median classification performance for ten random combinations of two groups of pretrained digits is $98.54 \%$, the $25^{\text {th }}$-percentile $97.26 \%$, and the $75^{\text {th }}$-percentile 99.5\% (Suppl. Table S1).

A detailed mathematical analysis (Methods) shows that heterosynaptic plas- 
ticity in equation (1) ensures that the total strength of the receptive field of each selective DGC converges to a stable value which is similar for selective DGCs. As a consequence, synaptic weights are intrinsically bounded without the need to impose hard bounds on the weight dynamics. Moreover, the spatial structure of the receptive field represents the weighted average of all those input patterns for which that DGC is responsive. The mathematical analysis also shows that those DGCs that do not develop selectivity have weak synaptic connections and a very low total strength of the receptive field.

\section{Newborn neurons become selective for novel patterns dur- ing maturation}

After convergence of synaptic weights during pretraining, selective DGCs are considered mature cells. Some DGCs did not develop any strong weight patterns and exhibit unselective receptive fields after pretraining (highlighted in red in Fig. 17). We classify these as unresponsive units. Since unresponsive model units have weak synaptic connections, we assume them to die because of lack of NMDA receptor activation (Tashiro et al., 2006), and replace them in the model by plastic newborn DGCs. Mature cells are less plastic than newborn cells (Schmidt-Hieber et al., 2004; Ge et al., 2007), so we set $\eta=0$ in equation (1) for mature cells and $\eta=0.01$ for newborn cells. Feedforward connection weights from EC to mature cells remain therefore fixed in our model. To mimic exposure of an animal to a novel set of stimuli, we now add input patterns from digit 5 to the set of presented stimuli, which was previously limited to patterns of digits 3 and 4 .

We postulate that functional integration of newborn DGCs requires the twostep maturation process caused by the GABA-switch from excitation to inhibition. Since excitatory GABAergic input potentially increases correlated activity within the dentate gyrus network, we predict that newborn DGCs respond to familiar stimuli during the early phase of maturation, but not during the late phase, when inhibitory GABAergic input leads to competition.

To test this hypothesis, our model newborn DGCs go through two maturation phases (Methods). The early phase of maturation is cooperative because, for each pattern presentation, activated mature DGCs indirectly excite the newborn DGCs via GABAergic interneurons. We assume that in natural settings, this GABAergic activation stays below the reversal potential of the GABA channels at which shunting inhibition would be induced (Heigele et al., 2016). This lateral activation of newborn DGCs drives the growth of their receptive fields in a direction similar to those of the currently active mature DGCs. Consistent with our hypothesis we find that, at the end of the early phase of maturation, newborn DGCs show a receptive field corresponding to a mixture of several input patterns (Fig. 2a). 
In the late phase of maturation, model newborn DGCs receive inhibitory GABAergic input from interneurons, similar to the input received by mature DGCs. Given that at the end of the early phase, newborn DGCs have receptive fields similar to those of mature DGCs, lateral inhibition induces competition with mature DGCs for activation during presentation of patterns from the novel digit. Because model newborn DGCs start their late phase of maturation with a higher excitability (lower threshold) compared to mature DGCs, consistent with observed enhanced excitability of newborn cells (Schmidt-Hieber et al., 2004; Li et al., 2017), the activation of newborn DGCs is facilitated for those input patterns for which no mature DGC has preexisting selectivity. Therefore, in the late phase of maturation, competition drives the synaptic weights of newborn DGCs towards receptive fields corresponding to different subcategories of the ensemble of input patterns of the novel digit 5 (Fig. $2 \mathrm{~b}$ ).

During maturation, the L2-norm of the feedforward weight vector onto newborn DGCs increases (Fig. 2e) indicating an increase in total glutamatergic innervation, e.g. through an increase in the number and size of spines (Zhao et al., 2006). Nevertheless, the distribution of firing rates of newborn DGCs is shifted to lower values at the end of the late phase compared to the end of the early phase of maturation (Fig. 2k,d), consistent with in vivo calcium imaging recordings showing that adult-born DGCs are more active than mature DGCs (Danielson et al. 2016).

We emphasize that upon presentation of a pattern of a given digit, only those DGCs with a receptive field similar to the specific writing style of the presented pattern become strongly active, others fire at a medium firing rate, yet others at a low rate $($ Fig. $2 \mathrm{~s})$. As a consequence, the firing rate of a particular newborn DGC at the end of its maturation to a pattern from digit 5 is strongly modulated by the specific choice of stimulation pattern within the class of ' 5 's. Analogous results are obtained for patterns from pretrained digits 3 and 4 (Suppl. Fig S1). Hence, the ensemble of DGCs is effectively performing pattern separation within each digit class as opposed to a simple ternary classification task. The selectivity of newborn DGCs develops during maturation. Indeed, during the late, competitive, phase, the percentage of active newborn DGCs decreases, both upon presentation of familiar patterns (digits 3 and 4), as well as upon presentation of novel patterns (digit 5) (Fig. 2f). This reflects the development of the selectivity of our model newborn DGCs from broad to narrow tuning, consistent with experimental observations (Marín-Burgin et al., 2012; Danielson et al., 2016).

\section{Adult-born neurons promote better discrimination}

As above, we compute classification performance of our model network as a surrogate for behavioral discrimination (Woods et al., 2020). At the end of the late 
phase of maturation of newborn DGCs, we obtain an overall classification performance of $94.56 \%$ for the three ensembles of digits (classification performance for digit 3: 90.50\%; digit 4: 98.17\%; digit 5: 95.18\%). Confusion matrices show that although novel patterns are not well classified at the end of the early phase of maturation $(\overline{\text { Fig. } 3})$, they are as well classified as pretrained patterns at the end of the late phase of maturation (Fig. 3).

We compare this performance with that of a network where all three digit ensembles are simultaneously pretrained $($ Fig. 3 3 , control 1$)$. In this case, the overall classification performance is $92.09 \%$ (classification performance for digit 3 : 86.83\%; digit 4: 98.78\%; digit 5: 90.70\%). The confusion matrix show that all three digits are decently classified, but with an overall lower performance (Fig. 3d). Across ten simulation experiments, classification performance is significantly higher when a novel ensemble of patterns is learned sequentially by newborn DGCs, than if all patterns are learned simultaneously (Wilcoxon signed rank test: $\mathrm{p}$-val $=0.0020$, Wilcoxon signed rank $=55$; one-way t-test: $\mathrm{p}$-val $=$ 0.0269, t-stat $=2.6401, \mathrm{df}=9$; Suppl. Table S1).

Furthermore, if two novel ensembles of digits (instead of a single one) are introduced during maturation of newborn DGCs, we observe that some newborn DGCs become selective for one of the novel digits, while others become selective for the other novel digit (Suppl. Fig. S2a). This was expected, since we have found earlier that DGCs are becoming selective for different prototype writing styles even within a digit category; hence introducing several additional digit categories of novel patterns simply increases the prototype diversity. Therefore, newborn DGCs can ultimately promote separation of several novel overarching categories of patterns, no matter if they are learned simultaneously or sequentially (Suppl. Fig. S2b).

\section{The GABA-switch guides learning of novel representations}

To assess whether maturation of newborn DGCs promotes learning of a novel ensemble of digit patterns, we compare our results with a control model without neurogenesis (control 2). Similar to the neurogenesis case, patterns from the novel digit 5 are introduced after pretraining with patterns from digits 3 and 4 . The feedforward weights and thresholds of DGCs that developed selectivity during pretraining are fixed (learning rate $\eta=0$ ), while the thresholds and weights of all unresponsive neurons remain plastic after pretraining $(\eta=0.01)$. The only differences to the model with neurogenesis are that in the control case unresponsive neurons: (i) keep their feedforward weights (i.e., no reinitialization to low values), and (ii) keep the same connections from and to inhibitory neurons.

We find that without neurogenesis, the previously unresponsive DGCs do not 
become selective for the novel digit 5, no matter during how many epochs patterns are presented (we went up to 100 epochs) (Fig. 3b, control 2). Therefore, if patterns from digit 5 are presented to the network, the model fails to discriminate them from the previously learned digits 3 and 4: the overall classification performance is $81.69 \%$ (classification performance for digit 3: $85.94 \%$; digit 4 : 97.56\%; digit 5: $59.42 \%$ ). This result suggests that integration of newborn DGCs is beneficial for sequential learning of novel patterns.

As a further control (control 3), we compare with a model where all DGCs keep plastic feedforward weights at the end of pretraining and upon introduction of the novel digit 5, no matter if they became selective or not for the pretrained digits 3 and 4 . We observe that in the case where all neurons are plastic, learning of the novel digit occurs at the cost of loss of selectivity of mature neurons. Several DGCs switch their selectivity to become sensitive to the novel digit (Fig. 35), while none of the previously unresponsive units becomes selective for presented patterns (compare with Fig. 1k). In contrast to the model with neurogenesis, we observe a drop in classification performance to $90.92 \%$ (classification performance for digit 3: $85.45 \%$; digit 4: $98.37 \%$; digit 5: $88.90 \%$ ). We find that the classification performance for digit 3 is the one which decreases the most. This is due to the fact that many DGCs previously selective for digit 3 modified their weights to become selective for digit 5 . Importantly, the more novel patterns are introduced, the more overwriting of previously stored memories occurs. Hence, if all DGCs remain plastic, discrimination between a novel pattern and a familiar pattern stored long ago is impaired.

\section{Maturation of newborn neurons shapes the representation of novel patterns}

Since each input pattern stimulates slightly different, yet overlapping, subsets of the 100 model DGCs in a sparse code such that about 20 DGCs respond to each pattern (Fig. 2 $\mathrm{g}$ ), there is no simple one-to-one assignment between neurons and patterns. In order to visualize the activity patterns of the ensemble of DGCs, we perform dimensionality reduction. We construct a two-dimensional space using the activity patterns of the network at the end of the late phase of maturation of newborn DGCs trained with '3's, '4's and '5's. One axis connects the center of mass (in the 100-dimensional activity space) of all DGC responses to '3's with all responses to '5's (arbitrarily called 'axis 1') and the other axis those from '4's to '5's (arbitrarily called 'axis 2'). We then project the activity of the 100 model DGCs upon presentation of MNIST testing patterns onto those two axes, both at the end of the early and late phase of maturation of newborn DGCs (Methods). Each 2-dimensional projection is illustrated by a dot whose color corresponds to the digit class of the presented input pattern (blue for digit 3, green for digit 4, 
red for digit 5). Different input patterns within the same digit class cause different activation patterns of the DGCs, as depicted by extended clouds of dots of the same color (Fig. 4a,b). Interestingly, an example pattern of a '5' that is visually similar to a '4' (characterized by the green cross) yields a DGC representation that lies closer to other '4's (green cloud of dots) than to typical '5's (red cloud of dots) (Fig. 4b). Noteworthy the separation of the representation of '5's from '3's and ' 4 's is better at end of the late phase (Fig. $4 \mathrm{~b})$ when compared to the end of the early phase of maturation $($ Fig. 4 a $)$. For instance, even though the pattern ' 5 ' corresponding to the orange cross is represented close to representations of ' 4 's at the end of the early phase of maturation (green cloud of dots, Fig. 4a), it is represented far from any '3's and '4's at the end of maturation (Fig. 4b). The expansion of the representation of ' 5 's into a previously empty subspace evolves as a function of time during the late phase of maturation (Fig. 4 d ).

\section{Robustness of the model}

Our results are robust to changes in network architecture. As mentioned earlier, neither the exact number of GABAergic neurons (Suppl. Table S2), nor that of DGCs is critical. Indeed, a larger network with 700 DGCs, thus mimicking the anatomically observed expansion factor of about 5 between EC and dentate gyrus (all other parameters unchanged), yields similar results (Suppl. Table S3).

In the network with 700 DGCs, 275 cells remain unresponsive after pretraining with digits 3 and 4 . In line with our earlier approach in the network with 100 DGCs, we can algoritmically replace all unresponsive neurons with newborn DGCs before patterns of digit 5 are added. Upon maturation, newborn DGC receptive fields provide a detailed representation of the prototypes of the novel digit 5 (Suppl. Fig. S4) and good classification performance is obtained (Suppl. Table S3). Interestingly, due to the randomness of the recurrent connections, some newborn DGCs become selective for particular prototypes of the familiar (pretrained) digits that are not already extensively represented by the network (see newborn DGCs selective for digit 4 highlighted by magenta squares in Suppl. Fig. S4).

As an alternative to replacing all unresponsive cells simultaneously, we can also replace only a fraction of them by newborn cells so as to simulate a continuous turn-over of cells. For example, if 119 of the 275 unresponsive cells are replaced by newborn DGCs before the start of presentations of digit 5, then these 119 cells become selective for different writing styles and generic features of the novel digit 5 (Suppl. Fig. S5) and allow a good classification performance of all three digits. On the other hand, replacing only 35 of the 275 unresponsive cells is not sufficient (Suppl. Table S3). In an even bigger network with more than 144 EC cells and more than 700 DGCs, we could choose to replace $1 \%$ of the total 
DGC population per week by newborn cells, consistent with biology (Van Praag et al., 1999; Cameron and McKay, 2001). Importantly, if only a small fraction of unresponsive cells are replaced at a given moment, other unresponsive cells remain available to be replaced later by newborn DGCs that are then ready to learn new stimuli.

Interestingly, the timing of the introduction of the novel stimulus is important. In our standard neurogenesis model, we introduce the novel digit 5 at the beginning of the early phase of maturation, which consists in one epoch of MNIST training patterns (all patterns are presented once). For the network with 100 DGCs, if the novel digit is only introduced in the middle of the early phase (half epoch), it cannot be properly learned (classification performance for digit 5: 46.52\%). However, if introduced after three-eights or one-quarter of the early phase, the novel digit can be picked out (classification performance for digit 5: $93.61 \%$ and $94.17 \%$ resp.). We thus observe an increase in performance the earlier the novel digit is introduced (classification performance for digit 5 was $95.18 \%$ when introduced at the beginning of the early phase of maturation). Therefore our model predicts that a novel stimulus has to be introduced early enough with respect to newborn DGC maturation to be well discriminated, and that the accuracy of discrimination is better the earlier it is introduced. This could lead to an online scenario of our model, where adult-born DGCs are produced every day and different classes of novel patterns are introduced at different timepoints. Then different model newborn DGCs would become selective for different novel patterns according to their maturation stage with respect to presentation of the novel patterns.

\section{Newborn dentate granule cells become selective for similar novel patterns}

To investigate whether our theory for integration of newborn DGCs can explain why adult dentate gyrus neurogenesis promotes discrimination of similar stimuli, but does not affect discrimination of distinct patterns (Clelland et al., 2009, Sahay et al. 2011a), we use a simplified competitive winner-take-all network (Methods). It contains only as many DGCs as trained clusters, and the GABAergic inhibitory neurons are implicitly modeled through direct DGC-to-DGC inhibitory connections. DGCs are either silent or active (binary activity state, while in the detailed network DGCs had continuous firing rates). The synaptic plasticity rule is however the same as for the detailed network, with different parameter values (Methods). We also construct an artificial data set (Fig. 5a,b) that allows us to control the similarity $s$ of pairs of clusters (Methods). The MNIST data set is not appropriate to distinguish similar from dissimilar patterns, because all digit clusters are similar and highly overlapping, reflected by a high within cluster dispersion (e.g. 
across the set of all ' 3 ') compared to the separation between clusters (e.g. typical '3' versus typical '5').

After a pretraining period, a first mature DGC responds to patterns of cluster 1 and a second mature DGC to those of cluster 2 (Fig. 5e,f). We then fix the feedforward weights of those two DGCs and introduce a newborn DGC in the network. Thereafter, we present patterns from three clusters (the two pretrained ones, as well as a novel one), while the plastic feedforward weights of the newborn DGC are the only ones that are updated. We observe that the newborn DGC ultimately becomes selective for the novel cluster if it is $\operatorname{similar}(s=0.8)$ to the two pretrained clusters (Fig. 5i), but not if it is distinct $(s=0.2$, Fig. 5j). The selectivity develops in two phases. In the early phase of maturation of the newborn model cell, a pattern from the novel cluster that is similar to one of the pretrained clusters activates the mature DGC that has a receptive field closest to the novel pattern. The activated mature DGC drives the newborn DGC via lateral excitatory GABAergic connections to a firing rate where LTP is triggered at active synapses onto the newborn DGC. LTP also happens when a pattern from one of the pretrained clusters is presented. Thus, synaptic plasticity leads to a receptive field that reflects the average of all stimuli from all three clusters (Fig. 5s).

To summarize our findings in a more mathematical language, we characterize the receptive field of the newborn cell by the vector of its feedforward weights. Analogous to the notion of a firing rate vector that represents the set of firing rates of an ensemble of neurons, the feedforward weight vector represents the set of weights of all synapses projecting onto a given neuron (Fig. 1b). In the early phase of maturation, for similar clusters, the feedforward weight vector onto the newborn DGC grows in the direction of the center of mass of all three clusters (the two pretrained ones and the novel one), because for each pattern presentation one of the mature DGCs becomes active (compare Fig. 5g and Fig. 5k). However, if the novel cluster has a low similarity to pretrained clusters, patterns from the novel cluster do not activate any of the mature DGCs. Therefore the receptive field of the newborn cell reflects the average of stimuli from the two pretrained clusters only (compare Fig. 5h and Fig. 5l).

As a result of the different orientation of the feedforward weight vector onto the newborn DGC at the end of the early phase of maturation, two different situations arise in the late phase of maturation, when lateral GABAergic connections are inhibitory. If the novel cluster is similar to the pretrained clusters, the weight vector onto the newborn DGC at the end of the early phase of maturation lies at the center of mass of all the patterns across the three clusters. Thus it is closer to the novel cluster than the weight vector onto either of the mature DGCs (Fig. 5g). So if a novel pattern is presented, the newborn DGC wins the competition between the three DGCs, and its feedforward weight vector moves towards the center of 
mass of the novel cluster (Fig. 5). By contrast, if the novel cluster is distinct, the weight vector onto the newborn DGC at the end of the early phase of maturation is located at the center of mass of the two pretrained clusters (Fig. 5h). If a novel pattern is presented, no output unit is activated since their receptive fields are not similar enough to the input pattern. Therefore the newborn DGC always stays silent and does not update its feedforward weights (Fig. 5j). These results are consistent with studies that have suggested that dentate gyrus is only involved in the discrimination of similar stimuli, but not distinct stimuli (Gilbert et al., 2001; Hunsaker and Kesner, 2008). For discrimination of distinct stimuli, another pathway might be used, such as the direct EC to CA3 connection (Yeckel and Berger, 1990; Fyhn et al., 2007).

In conclusion, our model suggests that adult dentate gyrus neurogenesis promotes discrimination of similar patterns because newborn DGCs can ultimately become selective for novel stimuli which are similar to already learned stimuli. On the other hand, newborn DGCs fail to represent novel distinct stimuli, precisely because they are too distinct from other stimuli already represented by the network. Presentation of novel distinct stimuli in the late phase of maturation therefore does not induce synaptic plasticity of the newborn DGC feedforward weight vector toward the novel stimuli. In the simplified network, the transition between similar and distinct can be determined analytically (Methods). This analysis clarifies the importance of the switch from cooperative dynamics (excitatory interactions) in the early phase to competitive dynamics (inhibitory interactions) in the late phase of maturation.

\section{Upon successful integration the receptive field of a newborn DGC represents an average of novel stimuli}

With the simplified model network, it is possible to analytically compute the maximal strength of the DGC receptive field via the L2-norm of the feedforward weight vector onto the newborn DGC (Suppl. Material). In addition, the angle between the center of mass of the novel patterns and the feedforward weight vector onto the adult-born DGC can also be analytically computed (Suppl. Material). To illustrate the analytical results and characterize the evolution of the receptive field of the newborn DGC, we thus examine the angle $\phi$ of the feedforward weight vector with the center of mass of the novel cluster (i.e. the average of the novel stimuli), as a function of maturation time (Fig. 6b,c and Suppl. Fig. S3).

In the early phase of maturation, the feedforward weight vector onto the newborn DGC grows, while its angle with the center of mass of the novel cluster stays constant (Suppl. Fig. S3). In the late phase of maturation, the angle $\phi$ between the center of mass of the novel cluster and the feedforward weight vector onto the 
newborn DGC decreases in the case of similar patterns (Fig. 6c, Suppl. Fig. S3), but not in the case of distinct patterns (Suppl. Fig. S3), indicating that the newborn DGC becomes selective for the novel cluster for similar but not for distinct patterns.

The analysis of the simplified model thus leads to a geometric picture that helps us to understand how the similarity of patterns influences the evolution of the receptive field of the newborn DGC before and after the switch from excitation to inhibition of the GABAergic input. For novel patterns that are similar to known patterns, the receptive field of a newborn DGC at the end of maturation represents the average of novel stimuli.

\section{Discussion}

While experimental studies, such as manipulating the ratio of NKCC1 to KCC2, suggest that the switch from excitation to inhibition of the GABAergic input onto adult-born DGCs is crucial for their integration into the preexisting circuit $(\mathrm{Ge}$ et al., 2006; Alvarez et al., 2016) and that adult dentate gyrus neurogenesis promotes pattern separation (Clelland et al., 2009; Sahay et al., 2011a; Jessberger et al., 2009), the link between channel properties and behavior has remained puzzling (Sahay et al., 2011b; Aimone et al., 2011). Our modeling work shows that the GABA-switch enables newborn DGCs to become selective for novel stimuli which are similar to familiar, already stored, representations, consistent with the experimentally-observed function of pattern separation (Clelland et al., 2009; Sahay et al., 2011a; Jessberger et al., 2009).

Previous modeling studies already suggested that newborn DGCs integrate novel inputs into the representation in dentate gyrus (Chambers et al., 2004; Becker, 2005; Crick and Miranker, 2006; Wiskott et al., 2006; Chambers and Conroy, 2007; Appleby and Wiskott, 2009; Aimone et al., 2009; Weisz and Argibay, 2009, 2012; Temprana et al., 2015; Finnegan and Becker, 2015; DeCostanzo et al., 2019). However, our work differs from them in four important aspects. First of all, we implement an unsupervised biologically plausible plasticity rule, while many studies used supervised algorithmic learning rules (Chambers et al., 2004; Becker, 2005; Chambers and Conroy, 2007; Weisz and Argibay, 2009; Finnegan and Becker, 2015; DeCostanzo et al., 2019). Second, as we model the formerly neglected GABA-switch, the connection weights from EC to newborn DGCs are grown from small values through cooperativity in the early phase of maturation. This integration step was mostly bypassed in earlier models by initialization of the connectivity weights towards newborn DGCs to random, yet fully grown values (Crick and Miranker, 2006; Aimone et al., 2009; Weisz and Argibay, 2009, 2012; Finnegan and Becker, 2015). Third, as the dentate gyrus network is com- 
monly modeled as a competitive network, weight normalization is crucial. In our framework, competition occurs during the late phase of maturation. Previous modeling works either applied algorithmic weight normalization or hard bounds on the weights at each iteration step (Crick and Miranker, 2006; Aimone et al., 2009: Weisz and Argibay, 2009, 2012; Temprana et al., 2015; Finnegan and Becker, 2015). Instead, our plasticity rule includes heterosynaptic plasticity which intrinsically softly bounds connectivity weights by a homeostatic effect. Finally, although some earlier computational models of adult dentate gyrus neurogenesis could explain the pattern separation abilities of newborn cells, separation was obtained independently of the similarity between the stimuli. Contrarily to experimental data, no distinction was made between similar and distinct patterns (Chambers et al., 2004; Becker, 2005; Crick and Miranker, 2006; Wiskott et al., 2006; Chambers and Conroy, 2007; Aimone et al., 2009; Appleby and Wiskott, 2009; Weisz and Argibay, 2012; Temprana et al., 2015; Finnegan and Becker, 2015; DeCostanzo et al., 2019). To our knowledge, we present the first model that can explain both: (i) how adult-born DGCs integrate into the preexisting network, and (ii) why they promote pattern separation of similar stimuli and not distinct stimuli.

Our work emphasizes why a two-phase maturation of newborn DGCs is beneficial for proper integration in the preexisting network. From a computational perspective, the early phase of maturation, when GABAergic inputs onto newborn DGCs are excitatory, corresponds to cooperative unsupervised learning. Therefore, the synapses grow in the direction of patterns that indirectly activate the newborn DGCs via GABAergic interneurons (Fig. 6a). At the end of the early phase of maturation, the receptive field of a newborn DGC represents the center of mass of all input patterns that led to its (indirect) activation. In the late phase of maturation, GABAergic inputs onto newborn DGCs become inhibitory, so that lateral interactions change from cooperation to competition, causing a shift of the receptive fields of the newborn DGCs towards novel features (Fig. 6p). At the end of maturation, newborn DGCs are thus selective for novel inputs. This integration mechanism is in agreement with the experimental observation that newborn DGCs are broadly tuned early in maturation, yet highly selective at the end of maturation (Marín-Burgin et al., 2012; Danielson et al., 2016). Loosely speaking, the cooperative phase of excitatory GABAergic input promotes the growth of the synaptic weights coarsely in the relevant direction, whereas the competitive phase of inhibitory GABAergic input helps to specialize on detailed, but potentially important differences between patterns.

In the context of theories of unsupervised learning, the switch of lateral GABAergic input to newborn DGCs from excitatory to inhibitory provides a biological solution to the "problem of unresponsive units" (Hertz et al., 1991). Unsupervised competitive learning has been used to perform clustering of input patterns into a few categories (Rumelhart and Zipser, 1985, Grossberg, 1987; Kohonen, 1989; Hertz et al., 1991; Du, 2010). Ideally, after learning of the feedforward 
weights between an input layer and a competitive network, input patterns that are distinct from each other activate different neuron assemblies of the competitive network. After convergence of competitive Hebbian learning, the vector of feedforward weights onto a given neuron points to the center of mass of the cluster of input patterns for which it is selective (Kohonen, 1989; Hertz et al., 1991). Yet, if the synaptic weights are randomly initialized, it is possible that the set of feedforward weights onto some neurons of the competitive network point in a direction "quasi-orthogonal" (Methods) to the subspace of the presented input patterns. Therefore those neurons, called "unresponsive units", will never get active during pattern presentation. Different learning strategies have been developed in the field of artificial neural networks to avoid this problem (Grossberg, 1976; Bienenstock et al., 1982; Rumelhart and Zipser, 1985; Grossberg, 1987; DeSieno, 1988; Kohonen, 1989; Hertz et al., 1991; Du, 2010). However, most of these algorithmic approaches lack a biological interpretation. In our model, weak synapses onto newborn DGCs form spontaneously after neuronal birth. The excitatory GABAergic input in the early phase of maturation drives the growth of the synaptic weights in the direction of the subspace of presented patterns that succeed in activating some of the mature DGCs. Hence the early cooperative phase of maturation can be seen as a smart initialization of the synaptic weights onto newborn DGCs, close enough to novel patterns so as to become selective for them in the late competitive phase of maturation. However, the cooperative phase is helpful only if the novel patterns are similar to the input statistics defined by the set of known (familiar) patterns.

Our results are in line with the classic view that dentate gyrus is responsible for decorrelation of inputs (Marr, 1969; Albus, 1971; Marr, 1971; Rolls and Treves, 1998), a necessary step for differential storage of similar memories in CA3, and with the observation that dentate gyrus lesions impair discrimination of similar but not distinct stimuli (Gilbert et al., 2001; Hunsaker and Kesner, 2008). To discriminate distinct stimuli, another pathway might be involved, such as the direct EC to CA3 connection (Yeckel and Berger, 1990; Fyhn et al., 2007).

Our model of transition from an early cooperative phase to a late competitive phase makes specific predictions, at the behavioral and cellular level. In our model, the early cooperative phase of maturation can only drive the growth of synaptic weights onto newborn cells if they are indirectly activated by mature DGCs through GABAergic input, which has an excitatory effect due to the high NKCC1/KCC2 ratio early in maturation. Therefore our model predicts that NKCC1-knockout mice would be impaired in discriminating similar contexts or objects because newborn cells stay silent due to lack of indirect activation. The feedforward weight vector onto newborn DGCs could not grow in the early phase and newborn DGCs could not become selective for novel inputs. Therefore our model predicts that since newborn DGCs are poorly integrated into the preexisting circuit, they are unlikely to survive. If, however, in the same paradigm 
newborn cells are activated by light-induced or electrical stimulation, we predict that they become selective to novel patterns. Thus discrimination abilities would be restored and newborn DGCs are likely to survive. Analogously, we predict that using inducible NKCC1-knockout mice, animals would gradually be impaired in discrimination tasks after induced knockout and reach a stable maximum impairment about 3 weeks after the start of induced knockout.

Experimental observations support the importance of the switch from early excitation to late inhibition of the GABAergic input onto newborn DGCs. An absence of early excitation using NKCC1-knockout mice has been shown to strongly affect synapse formation and dendritic development in vivo (Ge et al., 2006). Conversely, a reduction in inhibition in the dentate gyrus through decrease in KCC2 expression has been associated with epileptic activity (Pathak et al., 2007; Barmashenko et al. 2011). An analogous switch of the GABAergic input has been observed during development, and its proper timing has been shown to be crucial for sensorimotor gating and cognition Wang and Kriegstein, 2010; Furukawa et al., 2017). In addition to early excitation and late inhibition, our theory also critically depends on the switch. In our model, the switch makes an instantaneous transition between early and late phase of maturation. Several experimental results have suggested that the switch is indeed sharp and occurs within a single day, both during development (Khazipov et al., 2004; Tyzio et al., 2007; Leonzino et al., 2016) and adult dentate gyrus neurogenesis (Heigele et al., 2016). Furthermore, in hippocampal cell cultures, expression of KCC2 is upregulated by GABAergic activity but not affected by glutamatergic activity (Ganguly et al., 2001). A similar process during adult dentate gyrus neurogenesis would increase the number of newborn DGCs available for representing novel features by advancing the timing of their switch. In this way, instead of a few thousands of newborn DGCs ready to switch (3 to $6 \%$ of the whole population (Van Praag et al., 1999; Cameron and McKay, 2001), divided by 30 days), a larger fraction of newborn DGCs would be made available for coding, if appropriate stimulation occurs.

To conclude, our theory for integration of adult-born DGCs suggests that newborn cells have a coding -rather than a modulatory- role during dentate gyrus pattern separation function. Our theory highlights the importance of GABAergic input in adult dentate gyrus neurogenesis, and links the switch from excitation to inhibition to the integration of newborn DGCs into the preexisting circuit. Finally, it illustrates how Hebbian plasticity of EC to DGC synapses along with the switch make newborn cells suitable to promote pattern separation of similar but not distinct stimuli, a long-standing mystery in the field of adult dentate gyrus neurogenesis (Sahay et al., 2011b; Aimone et al., 2011). 


\section{Methods}

\section{Network architecture and neuronal dynamics}

DGCs are the principal cells of the dentate gyrus. They mainly receive excitatory projections from the entorhinal cortex through the perforant path and GABAergic inputs from local interneurons, as well as excitatory input from Mossy cells. They project to CA3 pyramidal cells and inhibitory neurons, as well as local Mossy cells (Acsády et al., 1998; Henze et al., 2002, Amaral et al., 2007; Temprana et al. 2015). In our model, we omit Mossy cells for simplicity and describe the dentate gyrus as a competitive circuit consisting of $N_{D G C}$ dentate granule cells and $N_{I}$ GABAergic interneurons (Fig. 1b). The activity of $N_{E C}$ neurons in EC represents an input pattern $\vec{x}=\left(x_{1}, x_{2}, \ldots, x_{N_{E C}}\right)$. Because the perforant path also induces strong feedforward inhibition in the dentate gyrus (Li et al., 2013), we assume that the effective EC activity is normalized, such that $\|\vec{x}\|=1$ for any input pattern $\vec{x}$. We use $P$ different input patterns $\vec{x}^{\mu}, 1 \leqslant \mu \leqslant P$ in the simulations of the model.

In our network, model EC neurons have excitatory all-to-all connections to the DGCs. In rodent hippocampus, spiking mature DGCs activate interneurons in DG, which in turn inhibit other mature DGCs (Temprana et al., 2015, Alvarez et al., 2016). In our model, the DGCs are thus recurrently connected with inhibitory neurons (Fig. 1b). Connections from DGCs to interneurons exist in our model with probability $p_{I E}$ and have a weight $w_{I E}$. Similarly, connections from interneurons to DGCs occur with probability $p_{E I}$ and have a weight $w_{E I}$. All parameters are reported in Table 1(Biologically-plausible network).

Before an input pattern is presented, all rates of model DGCs are initialized to zero. Upon stimulation with input pattern $\vec{x}$, the firing rate $\nu_{i}$ of DGC $i$ evolves according to (Miller and Fumarola, 2012):

$$
\tau_{m} \frac{\mathrm{d} \nu_{i}}{\mathrm{~d} t}=-\nu_{i}+\tanh \left(\frac{\left[I_{i}-b_{i}\right]_{+}}{L}\right)
$$

where $[.]_{+}$denotes rectification: $[a]=a$ for $a>0$ and zero otherwise. Here, $b_{i}$ is a firing threshold, $L=0.5$ is the smoothness parameter of the frequency-current curve ( $L^{-1}$ is the slope of the frequency-current curve at the firing threshold), and $I_{i}$ the total input to cell $i$ :

$$
I_{i}=\sum_{j=1}^{N_{E C}} w_{i j} x_{j}+\sum_{k=1}^{N_{I}} w_{i k}^{E I} \nu_{k}^{I}
$$

with $x_{j}$ the activity of EC input neuron $j, w_{i j} \geqslant 0$ the feedforward weight from EC input neuron $j$ to DGC $i$, and $w_{i k}^{E I}$ the weight from inhibitory neuron $k$ to 
DGC $i$. The sum runs over all inhibitory neurons, but the weights are set to $w_{i k}^{E I}=0$ if the connection is absent. The firing rate $\nu_{i}$ is unit-free and normalized to a maximum of 1 , which we interpret as a firing rate of $10 \mathrm{~Hz}$. We take the synaptic weights as unit-less parameters such that $I_{i}$ is also unit-free.

The firing rate $\nu_{k}^{I}$ of inhibitory neuron $k$, is defined as:

$$
\tau_{\operatorname{inh}} \frac{\mathrm{d} \nu_{k}^{I}}{\mathrm{~d} t}=-\nu_{k}^{I}+\left[I_{k}^{I}-p^{*} N_{D G C}\right]_{+}
$$

with $p^{*}$ a parameter which relates to the desired ensemble sparsity, and $I_{k}^{I}$ the total input towards interneuron $k$, given as:

$$
I_{k}^{I}=\sum_{i=1}^{N_{D G C}} w_{k i}^{I E} \nu_{i}
$$

with $w_{k i}^{I E}$ the weight from DGC $i$ to inhibitory neuron $k$. (We set $w_{k i}^{I E}=0$ if the connection is absent.) The feedback from inhibitory neurons ensures a sparse activity of model DGCs for each pattern. With $p^{*}=0.1$ we find that more than 70 $\%$ of model DGCs are silent (firing rate $<1 \mathrm{~Hz}$ (Senzai and Buzsáki, 2017)) when an input pattern is presented, and less than $10 \%$ are highly active (firing rate $>9 \mathrm{~Hz}$ (Fig. 2k,d), consistent with the experimentally observed sparse activity in dentate gyrus (Chawla et al., 2005).

\section{Plasticity rule}

Projections from EC onto newborn DGCs exhibit Hebbian plasticity (SchmidtHieber et al., 2004, Ge et al., 2007; McHugh et al., 2007). Therefore, in our model the connections from EC neurons to DGCs are plastic, following a Hebbian learning rule which exhibits long-term depression (LTD) or long-term potentiation (LTP) depending on the firing rate $\nu_{i}$ of the postsynaptic cell (Bienenstock et al., 1982, Artola et al., 1990; Sjöström et al., 2001; Pfister and Gerstner, 2006). Input patterns $\vec{x}^{\mu}, 1 \leqslant \mu \leqslant P$, are presented in random order. For each input pattern, we let the firing rates converge for a time $T$ where $T$ was chosen long enough to achieve convergence to a precision of $10^{-6}$. After $n-1$ presentations (i.e. at time $(n-1) \cdot T)$ the weight vector has value $w_{i j}^{(n-1)}$. We then present the next pattern and update at time $n \cdot T\left(w_{i j}^{(n)}=w_{i j}^{(n-1)}+\Delta w_{i j}\right)$, according to the following plasticity rule:

$$
\Delta w_{i j}=\eta\left\{-\alpha x_{j} \nu_{i}\left[\theta-\nu_{i}\right]_{+}+\gamma x_{j} \nu_{i}\left[\nu_{i}-\theta\right]_{+}-\beta w_{i j}\left[\nu_{i}-\theta\right]_{+} \nu_{i}^{3}\right\}
$$

where $x_{j}$ is the firing rate of presynaptic EC input neuron $j, \nu_{i}$ the firing rate of postsynaptic DGC $i, \eta$ the learning rate, $\theta$ marks the transition from LTD to 
LTP, and the relative strength $\alpha, \gamma$ of LTP and LTD depend on $\theta$ via $\alpha=\frac{\alpha_{0}}{\theta^{3}}>0$ and $\gamma=\gamma_{0}-\theta>0$. The values of the parameters $\alpha_{0}, \gamma_{0}, \beta$, and $\theta$ are given in Table 1(Biologically-plausible network). The weights are hard-bounded from below at 0 , i.e. if equation (6) leads to a new weight smaller than zero, $w_{i j}$ is set to zero. The first two terms of equation (6) are a variation of the BCM rule (Bienenstock et al. 1982). The third term implements heterosynaptic plasticity (Chistiakova et al., 2014: Zenke and Gerstner, 2017). Because the first two terms of the plasticity rule are Hebbian and proportional to the presynaptic activity $x_{j}$, the active DGCs $\left(\nu_{i}>\theta\right)$ update their feedforward weights in direction of the input pattern $\vec{x}$. Moreover, all weights onto neuron $i$ are downregulated heterosynaptically by an amount that increases supra-linearly with the postsynaptic rate $\nu_{i}$. Similar to learning in a competitive network (Kohonen, 1989; Hertz et al., 1991), the vector of feedforward weights onto active DGCs will move towards the center of mass of the cluster of patterns they are selective for, as we will discuss now.

For a given input pattern $\vec{x}^{\mu}$, there are three fixed points for the postsynaptic firing rate: $\nu_{i}=0, \nu_{i}=\theta$, and $\nu_{i}=\hat{\nu}_{i}$ (the negative root is omitted, because $\nu_{i} \geqslant 0$ due to equation (2) . For $\nu_{i}<\theta$, there is LTD, so the weights move toward zero: $w_{i j} \rightarrow 0$, while for $\nu_{i}>\theta$, there is LTP, so the weights move toward $w_{i j} \rightarrow \frac{\gamma x_{j}^{\mu}}{\beta \hat{\nu}_{i}^{2}}$ (Fig. 1. $)$. The value of $\hat{\nu}_{i}$ is defined implicitly by the network equations (2) (5), If a pattern $\vec{x}^{\mu}$ is presented only for a short time these fixed points are not reached during a single pattern presentation.

\section{Winners, losers, and quasi-orthogonal inputs}

We define the winners as the DGCs which become strongly active $\left(\nu_{i}>\theta\right)$ during presentation of an input pattern. Since the input patterns are normalized to have an L2-norm of 1 ( $\left\|\vec{x}^{\mu}\right\|=1$ by construction), and the L2-norm of the feedforward weight vectors is bounded (see Section Direction and length of the weight vector), the winning units are the ones whose weight vectors $\vec{w}_{i}$ (row of the feedforward connectivity matrix) align best with the current input pattern $\vec{x}^{\mu}$. Furthermore, we say that an input pattern $\vec{x}^{\mu}$ is "quasi-orthogonal" to a weight vector $\vec{w}_{i}$ if $I_{i}=\sum_{j=1}^{N_{E C}} w_{i j} x_{j}+\sum_{k=1}^{N_{I}} w_{i k}^{E I} \nu_{k}^{I}<b_{i}$. If an input pattern $\vec{x}^{\mu}$ is quasi-orthogonal to a weight vector $\vec{w}_{i}$, then neuron $i$ does not fire in response to $\vec{x}^{\mu}$. Note that for a case without inhibitory neurons and with $b_{i} \rightarrow 0$, we recover the standard orthogonality condition.

\section{Direction and length of the weight vector}

Let us denote the ensemble of patterns for which neuron $i$ is a winner by $C_{i}$ and call this the set of winning patterns $\left(C_{i}=\left\{\mu \mid \nu_{i}>\theta\right\}\right)$. Suppose that neuron $i$ 
is quasi-orthogonal to all other patterns, so that for all $\mu \notin C_{i}$ we have $\nu_{i}=0$. Then the feedforward weight vector of neuron $i$ converges in expectation to:

$$
\vec{w}_{i}=\frac{\gamma}{\beta} \frac{\left\langle G_{1}\left(\nu_{i}\right) \vec{x}\right\rangle_{\mu \in C_{i}}}{\left\langle G_{2}\left(\nu_{i}\right)\right\rangle_{\mu \in C_{i}}}
$$

where $G_{1}\left(\nu_{i}\right)=\left(\nu_{i}-\theta\right) \nu_{i}$ and $G_{2}\left(\nu_{i}\right)=\left(\nu_{i}-\theta\right) \nu_{i}^{3}$. Hence $\vec{w}_{i}$ is a weighted average over all winning patterns.

The squared length of the feedforward weight vector can be computed by multiplying equation (7) with $\vec{w}_{i}$ :

$$
\left\|\vec{w}_{i}\right\|^{2}=\vec{w}_{i} \cdot \vec{w}_{i}=\frac{\gamma}{\beta} \frac{\left\langle G_{1}\left(\nu_{i}\right)\left(\vec{w}_{i} \cdot \vec{x}\right)\right\rangle_{\mu \in C_{i}}}{\left\langle G_{2}\left(\nu_{i}\right)\right\rangle_{\mu \in C_{i}}}
$$

Since input patterns have length one, the scalar product on the right-hand side can be rewritten as $\vec{w}_{i} \cdot \vec{x}=\left\|\vec{w}_{i}\right\| \cos (\alpha)$ where $\alpha$ is the angle between the weight vector and pattern $\vec{x}$. Division by $\left\|\vec{w}_{i}\right\|$ yields the L2-norm of the feedforward weight vector:

$$
\left\|\vec{w}_{i}\right\|=\frac{\gamma}{\beta} \frac{\left\langle G_{1}\left(\nu_{i}\right) \cos (\alpha)\right\rangle_{\mu \in C_{i}}}{\left\langle G_{2}\left(\nu_{i}\right)\right\rangle_{\mu \in C_{i}}}
$$

where the averages run, as before, over all winning patterns.

Let us now derive bounds for $\left\|\vec{w}_{i}\right\|$. First, since $\cos (\alpha) \leqslant 1$ we have $\left\langle G_{1}\left(\nu_{i}\right) \cos (\alpha)\right\rangle_{\mu \in C_{i}} \leqslant\left\langle G_{1}\left(\nu_{i}\right)\right\rangle_{\mu \in C_{i}}$. Second, since for all winning patterns $\nu_{i}>\theta$, where $\theta$ is the LTP threshold, we have $\left\langle G_{2}\left(\nu_{i}\right)\right\rangle_{\mu \in C_{i}} \geqslant\left\langle\left(\nu_{i}-\theta\right) \nu_{i}\right\rangle \theta^{2}$. Thus the length of the weight vector is finite and bounded by:

$$
\left\|\vec{w}_{i}\right\| \leqslant \frac{\gamma}{\beta} \frac{\left\langle G_{1}\left(\nu_{i}\right)\right\rangle_{\mu \in C_{i}}}{\left\langle G_{2}\left(\nu_{i}\right)\right\rangle_{\mu \in C_{i}}} \leqslant \frac{\gamma}{\beta} \frac{1}{\theta^{2}}
$$

It is possible to make the second bound tighter if we find the winning pattern with the smallest firing rate $\nu_{\min }$ such that $\nu_{i} \geqslant \nu_{\min } \forall i \in C_{i}$ :

$$
\left\|\vec{w}_{i}\right\| \leqslant \frac{\gamma}{\beta} \frac{1}{\left(\nu_{\min }\right)^{2}}
$$

The bound is reached if neuron $i$ is winner for a single input pattern.

We can also derive a lower bound. For a pattern $\mu \in C_{i}$, let us write the firing rate of neuron $i$ as $\nu_{i}(\mu)=\bar{\nu}_{i}+\Delta \nu_{i}(\mu)$ where $\bar{\nu}_{i}$ is the mean firing rate of neuron $i$ averaged across all winning patterns and $\left\langle\Delta \nu_{i}\right\rangle_{\mu \in C_{i}}=0$. We assume that the absolute size of $\Delta \nu_{i}$ is small, i.e., $\left\langle\left(\Delta \nu_{i}\right)^{2}\right\rangle_{\mu \in C_{i}} \ll\left(\bar{\nu}_{i}\right)^{2}$. Linearization of equation (9) around $\bar{\nu}_{i}$ yields:

$$
\left\|\vec{w}_{i}\right\|=\frac{\gamma}{\beta} \frac{G_{1}\left(\bar{\nu}_{i}\right)}{G_{2}\left(\bar{\nu}_{i}\right)}\langle\cos (\alpha)\rangle_{\mu \in C_{i}}+\frac{\gamma}{\beta} \frac{G_{1}^{\prime}\left(\bar{\nu}_{i}\right)}{G_{2}\left(\bar{\nu}_{i}\right)}\left\langle\cos (\alpha) \Delta \nu_{i}\right\rangle_{\mu \in C_{i}}
$$


Elementary geometric arguments for a neuron model with monotonically increasing frequency-current curve yield that the value of $\left\langle\cos (\alpha) \Delta \nu_{i}\right\rangle_{\mu \in C_{i}}$ is positive (or zero), because an increase in the angle $\alpha$ lowers both the cosine and the firing rate, giving rise to a positive correlation. Since we are interested in a lower bound, we can therefore drop the term proportional to $G_{1}^{\prime}$ and evaluate the ratio $G_{1} / G_{2}$ to find:

$$
\left\|\vec{w}_{i}\right\| \geqslant \frac{\gamma}{\beta} \frac{1}{\left(\bar{\nu}_{i}\right)^{2}}\langle\cos (\alpha)\rangle_{\mu \in C_{i}} \geqslant \frac{\gamma}{\beta} \frac{1}{\left(\nu_{\max }\right)^{2}} \cos (\hat{\alpha})
$$

where $\nu_{\max }$ is the maximal firing rate of a DGC and $\hat{\alpha}=\max _{\mu \in \mathrm{C}_{\mathrm{i}}}\{\alpha\}$ is the angle of the winning pattern that has the largest angle with the weight vector. The first bound is tight and is reached if neuron $i$ is winner for only two patterns.

To summarize we find that the length of the weight vector remains bounded in a narrow range. Hence, for a reasonable distribution of input patterns and weight vectors, the value of $\left\|\vec{w}_{i}\right\|$ is similar for different neurons $i$, so that the weight vector will have, after convergence, similar lengths for all DGCs that are winners for at least one pattern. In our simulations with the MNIST data set, we find that the length of feedforward weight vectors lies in the range between 9.3 and 11.1 across all responsive neurons with a mean value close to 10; cf. Fig. 2p.

\section{Early maturation phase}

During the early phase of maturation, the GABAergic input onto a newborn DGC with index $l$ has an excitatory effect. In the model, it is implemented as follows: $w_{l k}^{E I}=-w_{E I}>0$ with probability $p_{E I}$ for any interneuron $k$ and $w_{l k}^{E I}=0$ otherwise (no connection). Since newborn cells do not project yet onto inhibitory neurons (Temprana et al. 2015), we have $w_{k l}^{I E}=0 \forall l$. Newborn DGCs are known to have enhanced excitability (Schmidt-Hieber et al., 2004 Li et al., 2017), so their threshold is kept at $b_{l}=0 \forall l$. Because the newborn model DGCs receive lateral excitation via interneurons and their thresholds are zero during the early phase of maturation, the lateral excitatory GABAergic input is always sufficient to activate them. Hence, if the firing rate of a newborn DGC exceeds the LTP threshold $\theta$, the feedforward weights grow towards the presented input pattern, cf. equation (6),

Presentation of all patterns of the data set once (1 epoch) is sufficient to reach convergence of the feedforward weights onto newborn DGCs. We define the end of the first epoch as the end of the early phase, i.e., simulation of one epoch of the model corresponds to about three weeks of biological time. 


\section{Late maturation phase}

During the late phase of maturation (starting at about 3 weeks (Ge et al., 2006)), the GABAergic input onto newborn DGCs switches from excitatory to inhibitory. In terms of our model, it means that all existing $w_{l k}^{E I}$ connections switch their sign to $w_{E I}<0$. Furthermore, since newborn DGCs develop lateral connections to inhibitory neurons in the late maturation phase (Temprana et al., 2015), we set $w_{k l}^{I E}=w_{I E}$ with probability $p_{I E}$, and $w_{k l}^{I E}=0$ otherwise. The thresholds of newborn DGCs are updated after presentation of pattern $\mu$ at time $n \cdot T\left(b_{l}^{(n)}=\right.$ $\left.b_{l}^{(n-1)}+\Delta b_{l}\right)$ according to $\Delta b_{l}=\eta_{b}\left(\nu_{l}-\nu_{0}\right)$, where $\nu_{0}$ is a reference rate and $\eta_{b}$ a learning rate, to mimic the decrease of excitability as newborn DGCs mature (Table 1, Biologically-plausible network). Therefore the distribution of firing rates of newborn DGCs is shifted to the left (towards lower firing rates) at the end of the late phase of maturation compared to the early phase of maturation (Fig. 2k,d). A sufficient condition for a newborn DGC to win the competition upon presentation of patterns of the novel cluster is that the scalar product between a pattern of the novel cluster and the feedforward weight vector onto the newborn DGC is larger than the scalar product between the pattern of the novel cluster and the feedforward weight vector onto any of the mature DGCs. Analogous to the early phase of maturation, presentation of all patterns of the data set once (1 epoch) is sufficient to reach convergence of the feedforward weights onto newborn DGCs. We therefore consider that the late phase of maturation has been finished after one epoch.

\section{Input patterns}

Two different sets of input patterns are used. Both data sets have a number $K$ of clusters and several thousands of patterns per cluster. As a first data set, we use the MNIST $12 \times 12$ patterns (LeCun et al., 1998) $\left(N_{E C}=144\right)$, normalized such that the L2-norm of each pattern is equal to 1 . The training set contains approximately 6000 patterns per digit, while the testing set contains about 1000 patterns per digit (Fig. 1d).

As a second data set, we use hand-made artificial patterns designed such that the distance between the centers of any two clusters, or in other words their pairwise similarity, is the same. All clusters lie on the positive quadrant of the surface of a hypersphere of dimension $N_{E C}-1$. The cluster centers are Walsh 
patterns shifted along the diagonal (Fig. 5b):

$$
\begin{aligned}
\vec{P}^{1} & =\frac{1}{c_{0}}(1+\xi, 1-\xi, 1+\xi, 1-\xi, \ldots, 1+\xi, 1-\xi, 1+\xi, 1-\xi) \\
\vec{P}^{2} & =\frac{1}{c_{0}}(1+\xi, 1+\xi, 1-\xi, 1-\xi, \ldots, 1+\xi, 1+\xi, 1-\xi, 1-\xi) \\
& \quad \ldots \\
\vec{P}^{K} & =\frac{1}{c_{0}}(1+\xi, 1+\xi, 1+\xi, 1+\xi, \ldots, 1-\xi, 1-\xi, 1-\xi, 1-\xi)
\end{aligned}
$$

with $|\xi|<1$ a parameter that determines the spacing between clusters. $c_{0}$ is a normalization factor to ensure that the center of mass of all clusters has an L2-norm of 1:

$$
c_{0}=\sqrt{N_{E C}\left(1+\xi^{2}\right)} .
$$

The number of input neurons $N_{E C}$ is $N_{E C}=2^{K}$. The scalar product, and hence the angle $\Omega$, between the center of mass of any pair of clusters $k$ and $l(k \neq l)$ is a function of $\xi($ Fig. $5 \mathrm{a})$ :

$$
\vec{P}^{k} \cdot \vec{P}^{l}=\frac{1}{1+\xi^{2}}=\cos (\Omega)
$$

We define the pairwise similarity $s$ of two clusters as: $s=1-\xi$. Highly similar clusters have a large $s$ due to the small distance between their centers (hence a small $\xi)$.

To make the artificial data set comparable to the MNIST $12 \times 12$ data set, we choose $K=7$, so $N_{E C}=128$, and we generate 6000 noisy patterns per cluster for the training set and 1000 other noisy patterns per cluster for the testing set. Since our noisy high-dimensional input patterns have to be symmetrically distributed around the centers of mass $\vec{P}^{k}$, yet lie on the hypersphere, we have to use an appropriate sampling method. The patterns $\vec{x}^{\mu(k)}$ of a given cluster $k$ with center of mass $\vec{P}^{k}$ are thus sampled from a Von Mises-Fisher distribution (Mardia and Jupp, 2009):

$$
\vec{x}^{\mu(k)} \sim\left(\sqrt{1-a^{2}}\right) \vec{\zeta}+a \vec{P}^{k}
$$

with $\vec{\zeta}$ an L2-normalized vector taken in the space orthogonal to $\vec{P}^{k}$. The vector $\vec{\zeta}$ is obtained by performing the singular-value decomposition of $\vec{P}^{k}\left(U \Sigma V^{*}=\vec{P}^{k}\right)$, and multiplying the matrix $U$ (after removing its first column), which corresponds to the left-singular vectors in the orthogonal space to $\vec{P}^{k}$, with a vector whose elements are drawn from the standard normal distribution. Then the L2-norm of the obtained pattern is set to 1 , so that it lies on the surface of the hypersphere. A rejection sampling scheme is used to obtain $a$ (Mardia and Jupp, 2009). The sample $a$ is kept if $\kappa a+\left(N_{E C}-1\right) \ln (1-\psi a)-c \geqslant \ln (u)$, with $\kappa$ a concentration parameter, $\psi=\frac{1-b}{1+b}, c=\kappa \psi+\left(N_{E C}-1\right) \ln \left(1-\psi^{2}\right)$, $u$ drawn from a uniform 
distribution $u \sim U[0,1], a=\frac{1-(1+b) z}{1-(1-b) z}, b=\frac{N_{E C}-1}{\sqrt{4 \kappa^{2}+\left(N_{E C}-1\right)^{2}}+2 \kappa}$, and $z$ drawn from a beta distribution $z \sim \mathcal{B} e\left(\frac{N_{E C}-1}{2}, \frac{N_{E C}-1}{2}\right)$.

The concentration parameter $\kappa$ characterizes the spread of the distribution around the center $\vec{P}^{k}$. In the limit where $\kappa \rightarrow 0$, sampling from the Von MisesFisher distribution becomes equivalent to sampling uniformly on the surface of the hypersphere, so the clusters become highly overlapping. In dimension $N_{E C}=128$, if $\kappa>10^{3}$ the probability of overlap between clusters is negligible. We use a value $\kappa=10^{4}$.

\section{Classification performance (readout network)}

It has been observed that classification performance based on DGC population activity is a good proxy for behavioral discrimination (Woods et al., 2020). Hence, to evaluate whether the newborn DGCs contribute to the function of the dentate gyrus network, we study classification performance. Once the feedforward weights have been adjusted upon presentation of many input patterns from the training set (Section Plasticity rule), we keep them fixed and determine classification on the test set using artificial readout units ( $\mathrm{RO})$.

To do so, the readout weights $\left(w_{k i}^{R O}\right.$ from model DGC $i$ to readout unit $k$ ) are initialized at random values drawn from a uniform distribution: $w_{k i}^{R O} \sim \sigma \mathcal{U}(0,1)$, with $\sigma=0.1$. The number of readout units, $N_{R O}$, corresponds to the number of learned classes. To adjust the readout weights, all patterns of the training data set that belong to the learned classes are presented one after the other. For each pattern $\vec{x}^{\mu}$, we let the firing rate of the DGCs converge (values at convergence: $\nu_{i}^{\mu}$ ). The activity of a readout unit $k$ is given by:

$$
\nu_{k}^{R O, \mu}=g\left(I_{k}^{R O, \mu}\right)=g\left(\sum_{i=1}^{N_{D G C}} w_{k i}^{R O} \nu_{i}^{\mu}\right)
$$

As we aim to assess the performance of the network of DGCs, the readout weights are adjusted by an artificial supervised learning rule. The loss function, which corresponds to the difference between the activity of the readout units and a one-hot representation of the corresponding pattern label (Hertz et al., 1991),

$$
L\left(W^{R O}\right)=\frac{1}{2} \sum_{k=1}^{N_{R O}}\left(L_{k}^{\mu}-\nu_{k}^{R O, \mu}\right)^{2}
$$

with $L_{k}^{\mu}$ the element $k$ of a one-hot representation of the correct label of pattern $\vec{x}^{\mu}$, is minimized by stochastic gradient descent:

$$
\Delta w_{k i}^{R O, \mu}=\eta\left(L_{k}^{\mu}-\nu_{k}^{R O, \mu}\right) g^{\prime}\left(I_{k}^{R O, \mu}\right) \nu_{i}^{\mu}
$$


The readout units have a rectified hyperbolic tangent frequency-current curve: $g(x)=\tanh \left(2[x]_{+}\right)$, whose derivative is: $g^{\prime}(x)=2\left(1-\left(\tanh \left(2[x]_{+}\right)\right)^{2}\right)$. We learn the weights of the readout units over 100 epochs of presentations of all training patterns with $\eta=0.01$, which is sufficient to reach convergence.

Thereafter, the readout weights are fixed. Each test set pattern belonging to one of the learned classes is presented once, and the firing rates of the DGCs are let to converge. Finally, the activity of the readout units $\nu_{k}^{R O, \mu}$ is computed and compared to the correct label $L_{k}^{\mu}$ of the presented pattern. If the readout unit with the highest activity value is the one that represents the class of the presented input pattern, the pattern is said to be correctly classified. Classification performance is given by the number of correctly classified patterns divided by the total number of test patterns of the learned classes.

\section{Control cases}

In our standard setting, patterns from a third digit are presented to a network that has previously only seen patterns from two digits. The question is whether neurogenesis helps when adding the third digit. We use several control cases to compare with the neurogenesis case. In the first control case, all three digits are learned in parallel (Fig. 3a, control 1). In the two other control cases, we either keep all feedforward connections towards the DGCs plastic (Fig. 3k, control 3), or fix the feedforward connections for all selective DGCs but keep unselective neurons plastic (as in the neurogenesis case) (Fig. 3b, control 2). However, in both instances, the DGCs do not mature in the two-step process induced by the GABA-switch that is part of our model of neurogenesis.

\section{Pretraining with two digits}

As we are interested by neurogenesis at the adult stage, we pretrain the network with patterns from two digits, such that it already stores some memories before neurogenesis takes place. To do so, we randomly initialize the weights from EC neurons to DGCs: they are drawn from a uniform distribution $\left(w_{i j} \sim U[0,1]\right)$. The L2-norm of the feedforward weight vector onto each DGC is then normalized to 1 , to ensure fair competition between DGCs during learning. Then we present all patterns from digits 3 and 4 in random order, as many times as needed for convergence of the weights. During each pattern presentation the firing rates of the DGCs are computed (Section Network architecture and neuronal dynamics) and their feedforward weights are updated according to our plasticity rule (Section Plasticity rule). We find that we need approximately 40 epochs for convergence of the weights, and use 80 epochs to make sure that all weights are stable. 
At the end of pretraining, our network is considered to correspond to an adult stage, because some DGCs are selective for prototypes of the pretrained digits (Fig. 1p).

\section{Projection on pairwise discriminatory axes}

To assess how separability of the DGC activation patterns develops during the late phase of maturation of newborn DGCs, we project the population activity onto axes which are optimized for pairwise discrimination (patterns from digit 3 versus patterns from digit 5, 4 versus 5 , and 3 vs 4 ). Those axes are determined using Fisher linear discriminant analysis (LDA), as explained below.

We determine the vector of DGC firing rates, $\vec{\nu}$, at the end of the late phase of maturation of newborn DGCs upon presentation of each pattern, $\vec{x}$, from digits 3, 4 and 5 of the training MNIST dataset. The mean activity in response to all training patterns $\mu$ from digit $m, \vec{\mu}_{m}=\frac{1}{N_{m}} \sum_{\mu \in m} \vec{\nu}^{\mu}$, is computed for each of the three digits $\left(N_{m}\right.$ is the number of training patterns of digit $\left.m\right)$. The pairwise Fisher linear discriminant is defined as the linear function $\vec{w}^{T} \vec{\nu}$ that maximizes the distance between the means of the projected activity in response to two digits (eg. $m$ and $n$ ), while normalizing for within-digit variability. The objective function to maximize is thus given as:

$$
J(w)=\frac{w^{T} S_{B} w}{w^{T} S_{W} w}
$$

with $S_{B}=\left(\vec{\mu}_{m}-\vec{\mu}_{n}\right)\left(\vec{\mu}_{m}-\vec{\mu}_{n}\right)^{T}$ the between-digit scatter matrix, and $S_{W}=$ $\Sigma_{m}+\Sigma_{n}$ the within-digit scatter matrix $\left(\Sigma_{m}\right.$ is the covariance matrix of the DGC activity in response to pattern of digit $m$, and $\Sigma_{n}$ is the covariance matrix of the DGC activity in response to pattern of digit $n$ ). It can be shown that the direction of the optimal discriminatory axis between digit $m$ and $n$ is given by the eigenvector of $S_{W}^{-1} S_{B}$ with the corresponding largest eigenvalue.

We arbitrarily set "axis 1" as the optimal discriminatory axis between digit 3 and digit 5, "axis 2" as the optimal discriminatory axis between digit 4 and digit 5, and "axis 3 " as the optimal discriminatory axis between digit 3 and digit 4. For each of the three discriminatory axes, we define its origin (ie. projection value of 0 ) as the location of the average projection of all training patterns of the three digits on the corresponding axis. Fig. 4 represents the projections of DGC activity upon presentation of testing patterns at the end of the early and late phase of maturation of newborn DGCs onto the above-defined axes. 


\section{Statistics}

In the main text, we present a representative example with three digits from the MNIST data set (3, 4 and 5). It is selected from a set of ten random combinations of three different digits. For each combination, one network is pretrained with two digits for 80 epochs. Then the third digit is added and neurogenesis takes place (one epoch of early phase of maturation, and one epoch of late phase of maturation). Furthermore another network is pretrained directly with the three digits for 80 epochs. Classification performance is reported for all combinations (Suppl. Table S1).

\section{Simplified rate network}

We use a toy network and the artificial data set to determine if our theory of integration of newborn DGCs can explain why adult dentate gyrus neurogenesis helps for the discrimination of similar, but not for distinct patterns.

The rate network described above is simplified as follows. We use $K$ dentate granule cells for $K$ clusters. Their firing rate $\nu_{i}$ is given by:

$$
\tau_{m} \frac{\mathrm{d} \nu_{i}}{\mathrm{~d} t}=-\nu_{i}+\mathcal{H}\left(I_{i}-b_{i}\right)
$$

where $\mathcal{H}$ is the Heaviside step function. As before, $b_{i}$ is the threshold, and $I_{i}$ the total input towards neuron $i$ :

$$
I_{i}=\sum_{j=1}^{N_{E C}} w_{i j} x_{j}+\sum_{k \neq j}^{N_{D G C}} w_{r e c} \nu_{k}
$$

with $x_{j}$ the input of presynaptic EC neuron $j, w_{i j}$ the feedforward weight between EC neuron $j$ and DGC $i$, and $\nu_{k}$ the firing rate of DGC $k$. Inhibitory neurons are modeled implicitly: each DGC directly connects to all other DGCs via inhibitory recurrent connections of value $w_{\text {rec }}<0$. During presentation of pattern $\vec{x}^{\mu}$, the firing rates of the DGCs evolve according to equation (22). After convergence, the feedforward weights are updated: $w_{i j}^{(\mu)}=w_{i j}^{(\mu-1)}+\Delta w_{i j}$. The synaptic plasticity rule is the same as before, see equation (6), but with the parameters reported in Table 1(Simple network). They are different from those of the biologicallyplausible network because we now aim for a single winning neuron for each cluster. Note that for an LTP threshold $\theta<1$ all active DGCs update their feedforward weights, because of the Heaviside function for the firing rate equation (22).

Assuming a single winner $i^{*}$ for each pattern presentation, the input equation (23) to the winner is:

$$
I_{i^{*}}=\vec{w}_{i^{*}} \cdot \vec{x}
$$


while the input to the losers is:

$$
I_{i}=\vec{w}_{i} \cdot \vec{x}+w_{\text {rec }}
$$

Therefore, two conditions need to be satisfied for a solution with a single winner:

$$
\vec{w}_{i^{*}} \cdot \vec{x}>b_{i}
$$

for the winner to actually be active, and:

$$
\vec{w}_{i} \cdot \vec{x}+w_{\text {rec }}<b_{i}
$$

to prevent non-winners to become active. The value of $b_{i}$ in the model is lower in the early phase than in the late phase of maturation to mimic enhanced excitability (Schmidt-Hieber et al., 2004; Li et al., 2017).

\section{Similar versus distinct patterns with the artificial data set}

Using the artificial data set with $|\xi|<1$ (equation (14)), the scalar product between the centers of mass of two different clusters, given by equation (16), satisfies: $0.5 \leqslant \frac{1}{1+\xi^{2}} \leqslant 1$. This corresponds to $0^{\circ} \leqslant \Omega \leqslant \Omega_{\max }=60^{\circ}$.

After stimulation with a pattern $\vec{x}$, it takes some time before the firing rates of the DGCs converge. We call two patterns "similar" if they activate, at least initially, the same output unit, while we consider two patterns as "distinct" if they do not activate the same output unit, not even initially. We now show that, with a large concentration parameter $\kappa$, patterns of different clusters are similar if $\xi<\sqrt{\frac{\left\|\vec{w}_{i}\right\|}{b_{i}}-1}$ and distinct if $\xi>\sqrt{\frac{\left\|\vec{w}_{i}\right\|}{b_{i}}-1}$.

We first consider a DGC $i$ whose feedforward weight vector has converged towards the center of mass of cluster $k$. If an input pattern $\vec{x}^{\mu(k)}$ from cluster $k$ is presented, it will receive the following initial input:

$$
I_{i}=\vec{w}_{i} \cdot \vec{x}^{\mu(k)}=\left\|\vec{w}_{i}\right\| \cdot\left\|\vec{x}^{\mu(k)}\right\| \cdot \cos \left(\vartheta_{\mathrm{kk}}\right)=\left\|\vec{w}_{i}\right\| \cdot \cos \left(\vartheta_{\mathrm{kk}}\right)
$$

where $\vartheta_{\mathrm{kk}}$ is the angle between the pattern $\vec{x}^{\mu(k)}$ and the center of mass $\vec{P}^{k}$ of the cluster to which it belongs. The larger the concentration parameter $\kappa$ for the generation of the artificial data set, the smaller the dispersion of the clusters, and thus the larger $\cos \left(\vartheta_{\mathrm{kk}}\right)$. If instead, an input pattern from cluster $l$ is presented, that same DGC will receive a lower initial input:

$$
I_{i}=\vec{w}_{i} \cdot \vec{x}^{\mu(l)}=\left\|\vec{w}_{i}\right\| \cdot\left\|\vec{x}^{\mu(l)}\right\| \cdot \cos \left(\vartheta_{\mathrm{kl}}\right) \approx \frac{\left\|\vec{w}_{i}\right\|}{1+\xi^{2}}
$$

The approximation holds for a small dispersion of the clusters (large concentration parameter $\kappa$ ). We note that there is no subtraction of the recurrent input 
yet, because output units are initialized with zero firing rate before each pattern presentation. By definition, similar patterns stimulate (initially) the same DGCs. A DGC can be active for two clusters only if its threshold is:

$$
b_{i}<\frac{\left\|\vec{w}_{i}\right\|}{1+\xi^{2}}
$$

Therefore, with a high concentration parameter $\kappa$, patterns of different clusters are similar if $\xi<\sqrt{\frac{\left\|\vec{w}_{i}\right\|}{b_{i}}-1}$, while patterns of different clusters are distinct if $\xi>\sqrt{\frac{\left\|\vec{w}_{i}\right\|}{b_{i}}-1}$

\section{Parameter choice}

The upper bound of the expected L2-norm of the feedforward weight vector towards the DGCs at convergence can be computed, see equation (11). With the parameters in Table 1(Simple network), the value is $\left\|\overrightarrow{\vec{w}_{i}}\right\| \leqslant 1.5$. Moreover, the input patterns for each cluster are highly concentrated, hence their angle with the center of mass of the cluster they belong to is close to 0 , so we have $\left\|\vec{w}_{i}\right\| \approx 1.5$. Therefore, at convergence, a DGC selective for a given cluster $k$ receives an input $I_{i^{*}}=\vec{w}_{i^{*}} \cdot \vec{x}^{\mu(k)} \approx 1.5$ upon presentation of input patterns $\vec{x}^{\mu(k)}$ belonging to cluster $k$. We choose $b_{i}=1.2$ to satisfy equation (26). Given $b_{i}$ the threshold value $\xi_{\text {thresh }}$ for which two clusters are similar (and above which two clusters are distinct) can be determined by equation (30): $\xi_{\text {thresh }}=0.5$. We created a handmade data set with $\xi=0.2$ for the case of similar clusters (therefore with similarity $s=0.8$ ), and a handmade data set with $\xi=0.8$ for the distinct case (hence with similarity $s=0.2)$.

Let us suppose that the weights of DGC $i$ have converged and made this cell respond to patterns of cluster $i$. If another DGC $k$ of the network is selective for cluster $k$, cell $i$ gets the input $I_{i}=\vec{w}_{i} \cdot \vec{x}^{\mu(k)}+w_{\text {rec }} \approx \frac{1.5}{1+\xi^{2}}+w_{\text {rec }}$ upon presentation of input patterns $\vec{x}^{\mu(k)}$ belonging to cluster $k \neq i$. Hence, to satisfy equation $(27)$, we need $w_{\text {rec }}<b_{i}-\max _{\xi}\left(\frac{1.5}{1+\xi^{2}}\right) \approx-0.24$. We set $w_{\text {rec }}=-1.2$.

Furthermore, a newborn DGC is born with a null feedforward weight vector so that at birth, its input consists only of the indirect excitatory input from mature DGCs which vanishes if all DGCs are quiescent and takes a value $I_{i}=-w_{\text {rec }}>0$ if a mature DGC responds to the input. For the feedforward weight vector to grow, the newborn cell $i$ needs to be active. This could be achieved through spontaneous activity which could be implemented by setting the intrinsic firing threshold at birth to a value $b_{\text {birth }}<0$. In this case a difference between similar and distinct patterns is not expected. Alternatively, activity of newborn cells can be achieved in the absence of spontaneous activity under the condition $-w_{\text {rec }}>b_{\text {birth }}$. For the 
simulations with the toy model, we set $b_{\text {birth }}=0.9$ which leads to weight growth in newborn cells for similar, but not distinct patterns.

\section{Neurogenesis with the artificial data set}

To save computation time, we initialize the feedforward weight vectors of two mature DGCs at two training patterns randomly chosen from the first two clusters, normalized such that they have an L2-norm of 1.5. We then present patterns from clusters 1 and 2, and let the feedforward weights evolve according to equation (6) until they reach convergence.

We thereafter fix the feedforward weights onto the two mature cells, and introduce a novel cluster of patterns as well as a newborn DGC in the network. The sequence of presentation of patterns from the three clusters (a novel one and two pretrained ones) is random. The newborn DGC is born with a null feedforward weight vector, and its maturation follows the same rules as before (plastic feedforward weights). In the early phase, GABAergic input has an excitatory effect (Ge et al., 2006) and the newborn DGC does not inhibit the mature DGCs (Temprana et al. 2015). This is modeled by setting $w_{\text {rec }}^{N M}=-w_{\text {rec }}$ for the connections from mature to newborn DGC, and $w_{\mathrm{rec}}^{M N}=0$ for the connections from newborn to mature DGCs. The threshold of the newborn DGC starts at $b_{\text {birth }}=0.9$ at birth, mimicking enhanced excitability (Schmidt-Hieber et al., 2004; Li et al., 2017), and increases linearly up to 1.2 (same threshold as that of mature DGCs) over 12000 pattern presentations, reflecting loss of excitability with maturation. The exact time window is not critical. In the late phase of maturation of the newborn DGC, GABAergic input switches to inhibitory (Ge et al., 2006), and the newborn DGC recruits feedback inhibition onto mature DGCs (Temprana et al., 2015). It is modeled by switching the sign of the connection from mature to newborn DGC: $w_{\mathrm{rec}}^{N M}=w_{\mathrm{rec}}$, and establishing connections from newborn to mature DGCs: $w_{\text {rec }}^{M N}=w_{\text {rec}}$. Each of the 6000 patterns is presented once during the early phase of maturation, and once during the late phase of maturation.

The above paradigm is run separately for each of the two handmade data sets: the one where clusters are similar $(s=0.8)$, and the one where clusters are distinct $(s=0.2)$.

\section{Acknowledgments}

This research was supported by the Swiss National Science Foundation (no. 200020_184615) and by the European Union Horizon 2020 Framework Program under grant agreement no. 785907 (HumanBrain Project, SGA2). 
1112

1113

1114

1115

1116

1117

\section{Authors contributions}

O.G. developed the model and carried out the simulations. W.G. participated in discussions and helped designing the project. O.G. and W.G. wrote and validated the manuscript.

\section{Declaration of interests}

The authors declare no competing interests.

\section{References}

Acsády, L., Kamondi, A., Sík, A., Freund, T., and Buzsáki, G. (1998). GABAergic cells are the major postsynaptic targets of mossy fibers in the rat hippocampus. Journal of neuroscience, 18, 3386-3403.

Aimone, J. B., Deng, W., and Gage, F. H. (2011). Resolving new memories: a critical look at the dentate gyrus, adult neurogenesis, and pattern separation. Neuron, 70, 589-596.

Aimone, J. B., Wiles, J., and Gage, F. H. (2009). Computational influence of adult neurogenesis on memory encoding. Neuron, 61, 187-202.

Albus, J. (1971). A theory of cerebellar function. J. Mathematical Biosciences, $10,25-61$.

Alvarez, D. D., Giacomini, D., Yang, S. M., Trinchero, M. F., Temprana, S. G., Büttner, K. A., Beltramone, N., and Schinder, A. F. (2016). A disynaptic feedback network activated by experience promotes the integration of new granule cells. Science, 354, 459-465.

Amaral, D. G., Scharfman, H. E., and Lavenex, P. (2007). The dentate gyrus: fundamental neuroanatomical organization (dentate gyrus for dummies). Progress in brain research, 163, 3-22.

Andersen, P., Morris, R., Amaral, D., Bliss, T., and O’Keefe, J. eds. (2007). The hippocampus book. (Oxford university press).

Appleby, P. A. and Wiskott, L. (2009). Additive neurogenesis as a strategy for avoiding interference in a sparsely-coding dentate gyrus. Network: Computation in Neural Systems, 20, 137-161. 
Artola, A., Bröcher, S., and Singer, W. (1990). Different voltage dependent thresholds for inducing long-term depression and long-term potentiation in slices of rat visual cortex. Nature, 347, 69-72.

Babadi, B. and Sompolinsky, H. (2014). Sparseness and expansion in sensory representations. Neuron, 83, 1213-1226.

Barmashenko, G., Hefft, S., Aertsen, A., Kirschstein, T., and Köhling, R. (2011). Positive shifts of the $\mathrm{GABA}_{\mathrm{A}}$ receptor reversal potential due to altered chloride homeostasis is widespread after status epilepticus. Epilepsia, 52, 1570-1578.

Becker, S. (2005). A computational principle for hippocampal learning and neurogenesis. Hippocampus, 15, 722-738.

Ben-Ari, Y. (2002). Excitatory actions of GABA during development: the nature of the nurture. Nature Reviews Neuroscience, 3, 728-739.

Bienenstock, E. L., Cooper, L. N., and Munro, P. W. (1982). Theory for the development of neuron selectivity: Orientation specificity and binocular interaction in visual cortex. Journal of Neuroscience, 2, 32-48.

Cameron, H. A. and McKay, R. D. (2001). Adult neurogenesis produces a large pool of new granule cells in the dentate gyrus. Journal of Comparative Neurology, 435, 406-417.

Chambers, R. A. and Conroy, S. K. (2007). Network modeling of adult neurogenesis: shifting rates of neuronal turnover optimally gears network learning according to novelty gradient. Journal of cognitive neuroscience, 19, 1-12.

Chambers, R. A., Potenza, M. N., Hoffman, R. E., and Miranker, W. (2004). Simulated apoptosis/neurogenesis regulates learning and memory capabilities of adaptive neural networks. Neuropsychopharmacology, 29, 747-758.

Chancey, J. H., Adlaf, E. W., Sapp, M. C., Pugh, P. C., Wadiche, J. I., and Overstreet-Wadiche, L. S. (2013). GABA depolarization is required for experience-dependent synapse unsilencing in adult-born neurons. Journal of Neuroscience, 33, 6614-6622.

Chawla, M., Guzowski, J., Ramirez-Amaya, V., Lipa, P., Hoffman, K., Marriott, L., Worley, P., McNaughton, B., and Barnes, C. A. (2005). Sparse, environmentally selective expression of Arc RNA in the upper blade of the rodent fascia dentata by brief spatial experience. Hippocampus, 15, 579-586.

Chistiakova, M., Bannon, N., Bazhenov, M., and Volgushev, M. (2014). Heterosynaptic plasticity: Multiple mechanisms and multiple roles. The Neuroscientist, 20, 483-498. 
Clelland, C., Choi, M., Romberg, C., Clemenson, G., Fragniere, A., Tyers, P., Jessberger, S., Saksida, L., Barker, R., Gage, F., and Bussey, T. (2009). A functional role for adult hippocampal neurogenesis in spatial pattern separation. Science, 325, 210-213.

Crick, C. and Miranker, W. (2006). Apoptosis, neurogenesis, and information content in hebbian networks. Biological cybernetics, 94, 9-19.

Danielson, N. B., Kaifosh, P., Zaremba, J. D., Lovett-Barron, M., Tsai, J., Denny, C. A., Balough, E. M., Goldberg, A. R., Drew, L. J., Hen, R., Losonczy, A., and Kheirbek, M. A. (2016). Distinct contribution of adult-born hippocampal granule cells to context encoding. Neuron, 90, 101-112.

Dayer, A. G., Ford, A. A., Cleaver, K. M., Yassaee, M., and Cameron, H. A. (2003). Short-term and long-term survival of new neurons in the rat dentate gyrus. Journal of Comparative Neurology, 460, 563-572.

DeCostanzo, A. J., Fung, C. C. A., and Fukai, T. (2019). Hippocampal neurogenesis reduces the dimensionality of sparsely coded representations to enhance memory encoding. Frontiers in computational neuroscience, 12, 99.

Deng, W., Aimone, J. B., and Gage, F. H. (2010). New neurons and new memories: how does adult hippocampal neurogenesis affect learning and memory? Nature reviews neuroscience, 11, 339-350.

Deshpande, A., Bergami, M., Ghanem, A., Conzelmann, K.-K., Lepier, A., Götz, M., and Berninger, B. (2013). Retrograde monosynaptic tracing reveals the temporal evolution of inputs onto new neurons in the adult dentate gyrus and olfactory bulb. Proceedings of the National Academy of Sciences, 110, 11521161.

DeSieno, D. (1988). Adding a conscience to competitive learning. In IEEE international conference on neural networks, vol. 1, pp. 117-124. Institute of Electrical and Electronics Engineers New York.

Du, K.-L. (2010). Clustering: A neural network approach. Neural networks, 23, 89-107.

Finnegan, R. and Becker, S. (2015). Neurogenesis paradoxically decreases both pattern separation and memory interference. Frontiers in systems neuroscience, 9, 136 .

Freund, T. and Buzsáki, G. (1996). Interneurons of the hippocampus. Hippocampus, 6, 347-470. 
Furukawa, M., Tsukahara, T., Tomita, K., Iwai, H., Sonomura, T., Miyawaki, S., and Sato, T. (2017). Neonatal maternal separation delays the GABA excitatoryto-inhibitory functional switch by inhibiting $\mathrm{KCC} 2$ expression. Biochemical and biophysical research communications, 493, 1243-1249.

Fyhn, M., Hafting, T., Treves, A., Moser, M.-B., and Moser, E. I. (2007). Hippocampal remapping and grid realignment in entorhinal cortex. Nature, 446, 190-194.

Ganguly, K., Schinder, A. F., Wong, S. T., and Poo, M.-m. (2001). GABA itself promotes the developmental switch of neuronal GABAergic responses from excitation to inhibition. Cell, 105, 521-532.

Ge, S., Goh, E. L., Sailor, K. A., Kitabatake, Y., Ming, G.-l., and Song, H. (2006). GABA regulates synaptic integration of newly generated neurons in the adult brain. Nature, 439, 589-593.

Ge, S., Yang, C.-h., Hsu, K.-s., Ming, G.-l., and Song, H. (2007). A critical period for enhanced synaptic plasticity in newly generated neurons of the adult brain. Neuron, 54, 559-566.

Gilbert, P. E., Kesner, R. P., and Lee, I. (2001). Dissociating hippocampal subregions: A double dissociation between dentate gyrus and CA1. Hippocampus, 11, 626-636.

Groisman, A. I., Yang, S. M., and Schinder, A. F. (2020). Differential coupling of adult-born granule cells to parvalbumin and somatostatin interneurons. Cell reports, 30, 202-214.

Grossberg, S. (1976). Adaptive pattern classification and universal recoding II: Feedback, expectation, olfaction, illusions. Biological Cybernetics, 23, 187-202.

Grossberg, S. (1987). The Adaptive Brain I. (Elsevier).

Heigele, S., Sultan, S., Toni, N., and Bischofberger, J. (2016). Bidirectional GABAergic control of action potential firing in newborn hippocampal granule cells. Nature neuroscience, 19, 263-270.

Henze, D. A., Wittner, L., and Buzsáki, G. (2002). Single granule cells reliably discharge targets in the hippocampal CA3 network in vivo. Nature neuroscience, 5, 790-795.

Hertz, J., Krogh, A., and Palmer, R. G. (1991). Introduction to the Theory of Neural Computation. (Addison-Wesley).

Houser, C. R. (2007). Interneurons of the dentate gyrus: an overview of cell types, terminal fields and neurochemical identity. Progress in brain research, $163,217-232$. 
Hunsaker, M. R. and Kesner, R. P. (2008). Evaluating the differential roles of the dorsal dentate gyrus, dorsal CA3, and dorsal CA1 during a temporal ordering for spatial locations task. Hippocampus, 18, 955-964.

Jarrard, L. E. (1993). On the role of the hippocampus in learning and memory in the rat. Behavioral and neural biology, 60, 9-26.

Jessberger, S., Clark, R. E., Broadbent, N. J., Clemenson, G. D., Consiglio, A., Lie, D. C., Squire, L. R., and Gage, F. H. (2009). Dentate gyrus-specific knockdown of adult neurogenesis impairs spatial and object recognition memory in adult rats. Learning \& memory, 16, 147-154.

Johnston, S. T., Shtrahman, M., Parylak, S., Gonçalves, J. T., and Gage, F. H. (2016). Paradox of pattern separation and adult neurogenesis: A dual role for new neurons balancing memory resolution and robustness. Neurobiology of learning and memory, 129, 60-68.

Kee, N., Teixeira, C. M., Wang, A. H., and Frankland, P. W. (2007). Preferential incorporation of adult-generated granule cells into spatial memory networks in the dentate gyrus. Nature neuroscience, 10, 355-362.

Khazipov, R., Khalilov, I., Tyzio, R., Morozova, E., Ben-Ari, Y., and Holmes, G. L. (2004). Developmental changes in GABAergic actions and seizure susceptibility in the rat hippocampus. European Journal of Neuroscience, 19, 590-600.

Klausberger, T. and Somogyi, P. (2008). Neuronal diversity and temporal dynamics: the unity of hippocampal circuit operations. Science, 321, 53-57.

Kohonen, T. (1989). Self-organization and associative memory. (Springer-Verlag), 3rd edn.

LeCun, Y., Bottou, L., Bengio, Y., and Haffner, P. (1998). Gradient-based learning applied to document recognition. Proceedings of the IEEE, 86, 2278-2324.

Leonzino, M., Busnelli, M., Antonucci, F., Verderio, C., Mazzanti, M., and Chini, B. (2016). The timing of the excitatory-to-inhibitory GABA switch is regulated by the oxytocin receptor via KCC2. Cell reports, 15, 96-103.

Li, L., Sultan, S., Heigele, S., Schmidt-Salzmann, C., Toni, N., and Bischofberger, J. (2017). Silent synapses generate sparse and orthogonal action potential firing in adult-born hippocampal granule cells. Elife, 6, e23612.

Li, Y., Stam, F. J., Aimone, J. B., Goulding, M., Callaway, E. M., and Gage, F. H. (2013). Molecular layer perforant path-associated cells contribute to feedforward inhibition in the adult dentate gyrus. Proceedings of the National Academy of Sciences, 110, 9106-9111. 
Mardia, K. V. and Jupp, P. E. (2009). Directional statistics, vol. 494. (John Wiley \& Sons).

Marín-Burgin, A., Mongiat, L. A., Pardi, M. B., and Schinder, A. F. (2012). Unique processing during a period of high excitation/inhibition balance in adult-born neurons. Science, 335, 1238-1242.

Marr, D. (1969). A theory of cerebellar cortex. J. Physiology, 202, 437-470.

Marr, D. (1971). Simple memory: a theory for archicortex. Philosophical Transactions of the Royal Society of London, 262, 23-81.

McHugh, T. J., Jones, M. W., Quinn, J. J., Balthasar, N., Coppari, R., Elmquist, J. K., Lowell, B. B., Fanselow, M. S., Wilson, M. A., and Tonegawa, S. (2007). Dentate gyrus NMDA receptors mediate rapid pattern separation in the hippocampal network. Science, 317, 94-99.

Miller, K. D. and Fumarola, F. (2012). Mathematical equivalence of two common forms of firing rate models of neural networks. Neural computation, 24, 25-31.

Owens, D. F. and Kriegstein, A. R. (2002). Is there more to GABA than synaptic inhibition? Nature Reviews Neuroscience, 3, 715-727.

Pathak, H. R., Weissinger, F., Terunuma, M., Carlson, G. C., Hsu, F.-C., Moss, S. J., and Coulter, D. A. (2007). Disrupted dentate granule cell chloride regulation enhances synaptic excitability during development of temporal lobe epilepsy. Journal of Neuroscience, 27, 14012-14022.

Pfister, J.-P. and Gerstner, W. (2006). Triplets of spikes in a model of spike timing-dependent plasticity. Journal of Neuroscience, 26, 9673-9682.

Rolls, E. T. and Treves, A. (1998). Neural networks and brain function, vol. 572. (Oxford university press Oxford).

Rumelhart, D. E. and Zipser, D. (1985). Feature discovery by competitive learning. Cognitive science, 9, 75-112.

Sahay, A., Scobie, K. N., Hill, A. S., O'Carroll, C. M., Kheirbek, M. A., Burghardt, N. S., Fenton, A. A., Dranovsky, A., and Hen, R. (2011a). Increasing adult hippocampal neurogenesis is sufficient to improve pattern separation. Nature, 472, 466-470.

Sahay, A., Wilson, D. A., and Hen, R. (2011b). Pattern separation: a common function for new neurons in hippocampus and olfactory bulb. Neuron, 70, 582588. 
Schmidt-Hieber, C., Jonas, P., and Bischofberger, J. (2004). Enhanced synaptic plasticity in newly generated granule cells of the adult hippocampus. Nature, 429, 184-187.

Senzai, Y. and Buzsáki, G. (2017). Physiological properties and behavioral correlates of hippocampal granule cells and mossy cells. Neuron, 93, 691-704.

Sjöström, P., Turrigiano, G., and Nelson, S. (2001). Rate, timing, and cooperativity jointly determine cortical synaptic plasticity. Neuron, 32, 1149-1164.

Somogyi, P. and Klausberger, T. (2005). Defined types of cortical interneurone structure space and spike timing in the hippocampus. The Journal of physiology, $562,9-26$.

Stefanelli, T., Bertollini, C., Lüscher, C., Muller, D., and Mendez, P. (2016). Hippocampal somatostatin interneurons control the size of neuronal memory ensembles. Neuron, 89, 1074-1085.

Tashiro, A., Makino, H., and Gage, F. H. (2007). Experience-specific functional modification of the dentate gyrus through adult neurogenesis: a critical period during an immature stage. Journal of Neuroscience, 27, 3252-3259.

Tashiro, A., Sandler, V. M., Toni, N., Zhao, C., and Gage, F. H. (2006). NMDAreceptor-mediated, cell-specific integration of new neurons in adult dentate gyrus. Nature, 442, 929-933.

Temprana, S. G., Mongiat, L. A., Yang, S. M., Trinchero, M. F., Alvarez, D. D., Kropff, E., Giacomini, D., Beltramone, N., Lanuza, G. M., and Schinder, A. F. (2015). Delayed coupling to feedback inhibition during a critical period for the integration of adult-born granule cells. Neuron, 85, 116-130.

Tyzio, R., Holmes, G. L., Ben-Ari, Y., and Khazipov, R. (2007). Timing of the developmental switch in $\mathrm{GABA}_{\mathrm{A}}$ mediated signaling from excitation to inhibition in CA3 rat hippocampus using gramicidin perforated patch and extracellular recordings. Epilepsia, 48, 96-105.

Van Praag, H., Kempermann, G., and Gage, F. H. (1999). Running increases cell proliferation and neurogenesis in the adult mouse dentate gyrus. Nature neuroscience, 2, 266-270.

Vivar, C., Potter, M. C., Choi, J., Lee, J.-y., Stringer, T. P., Callaway, E. M., Gage, F. H., Suh, H., and Van Praag, H. (2012). Monosynaptic inputs to new neurons in the dentate gyrus. Nature Communications, 3, 1107.

Wang, D. D. and Kriegstein, A. R. (2010). Blocking early GABA depolarization with bumetanide results in permanent alterations in cortical circuits and sensorimotor gating deficits. Cerebral cortex, 21, 574-587. 
Wang, Y., Chik, D. T. W., and Wang, Z. D. (2000). Coherence resonance and noise-induced synchronization in globally coupled Hodgkin-Huxley neurons. Phys. Rev. E, 61, 740.

Weisz, V. I. and Argibay, P. F. (2009). A putative role for neurogenesis in neurocomputational terms: Inferences from a hippocampal model. Cognition, 112, 229-240.

Weisz, V. I. and Argibay, P. F. (2012). Neurogenesis interferes with the retrieval of remote memories: Forgetting in neurocomputational terms. Cognition, 125, $13-25$.

Wiskott, L., Rasch, M. J., and Kempermann, G. (2006). A functional hypothesis for adult hippocampal neurogenesis: avoidance of catastrophic interference in the dentate gyrus. Hippocampus, 16, 329-343.

Woods, N. I., Stefanini, F., Apodaca-Montano, D. L., Tan, I. M., Biane, J. S., and Kheirbek, M. A. (2020). The dentate gyrus classifies cortical representations of learned stimuli. Neuron, 107.

Yeckel, M. F. and Berger, T. W. (1990). Feedforward excitation of the hippocampus by afferents from the entorhinal cortex: redefinition of the role of the trisynaptic pathway. Proceedings of the National Academy of Sciences, 87, $5832-5836$.

Yuan, M., Meyer, T., Benkowitz, C., Savanthrapadian, S., Ansel-Bollepalli, L., Foggetti, A., Wulff, P., Alcami, P., Elgueta, C., and Bartos, M. (2017). Somatostatin-positive interneurons in the dentate gyrus of mice provide localand long-range septal synaptic inhibition. eLife, 6, e21105.

Zenke, F. and Gerstner, W. (2017). Hebbian plasticity requires compensatory processes on multiple timescales. Philosophical Transactions of the Royal Society B: Biological Sciences, 372, 1-17.

Zhao, C., Teng, E. M., Summers, R. G., Ming, G.-l., and Gage, F. H. (2006). Distinct morphological stages of dentate granule neuron maturation in the adult mouse hippocampus. Journal of Neuroscience, 26, 3-11. 

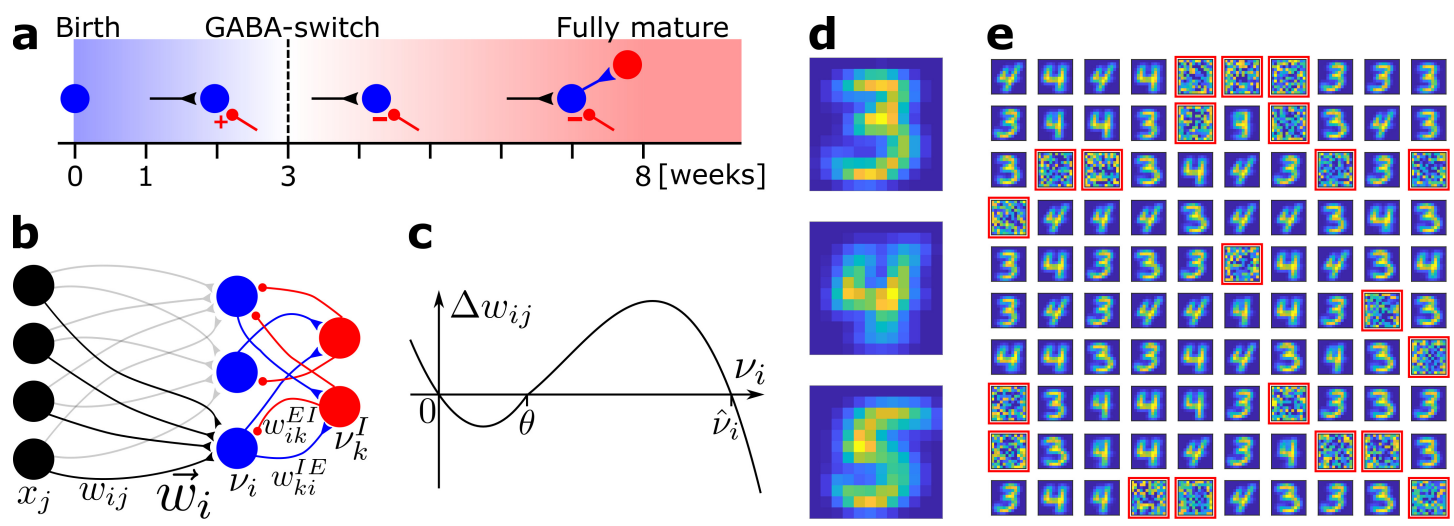

Figure 1: Network model and pretraining. (a) Integration of an adultborn DGC (blue) as a function of time: GABAergic synaptic input (red) switches from excitatory $(+)$ to inhibitory $(-)$; strong connections to interneurons develop only later; glutamatergic synaptic input (black), interneuron (red). (b) Network structure. EC neurons (black, rate $x_{j}$ ) are fully connected with weights $w_{i j}$ to DGCs (blue, rate $\nu_{i}$ ). The feedforward weight vector $\vec{w}_{i}$ onto neuron $i$ is depicted in black. DGCs and interneurons (red, rate $\nu_{k}^{I}$ ) are mutually connected with probability $p_{I E}$ and $p_{E I}$ and weights $w_{k i}^{I E}$ and $w_{i k}^{E I}$, respectively. Connections with a triangular (round) end are glutamatergic (GABAergic). (c) Given presynaptic activity $x_{j}>0$, the weight update $\Delta w_{i j}$ is shown as a function of the firing rate $\nu_{i}$ of the postsynaptic DGC with LTD for $\nu_{i}<\theta$ and LTP for $\theta<\nu_{i}<$ $\hat{\nu}_{i}$. (d) Center of mass for three ensembles of patterns from the MNIST data set, visualized as $12 \times 12$ pixel patterns. The two-dimensional arrangements and colors are for visualization only. (e) 100 receptive fields, each defined as the set of feedforward weights, are represented in a 2-dimensional organization. After pretraining with patterns from MNIST digits 3 and 4, 79 DGCs have receptive fields corresponding to threes and fours of different writing styles, while 21 remain unselective (highlighted by red frames). 


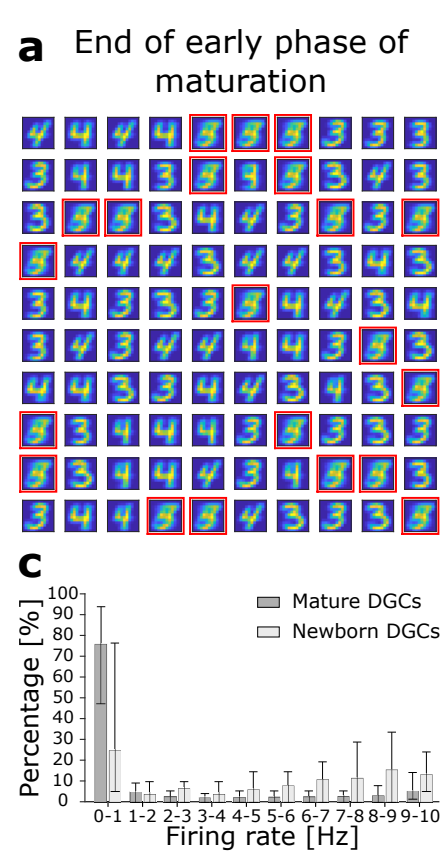

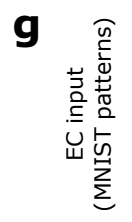
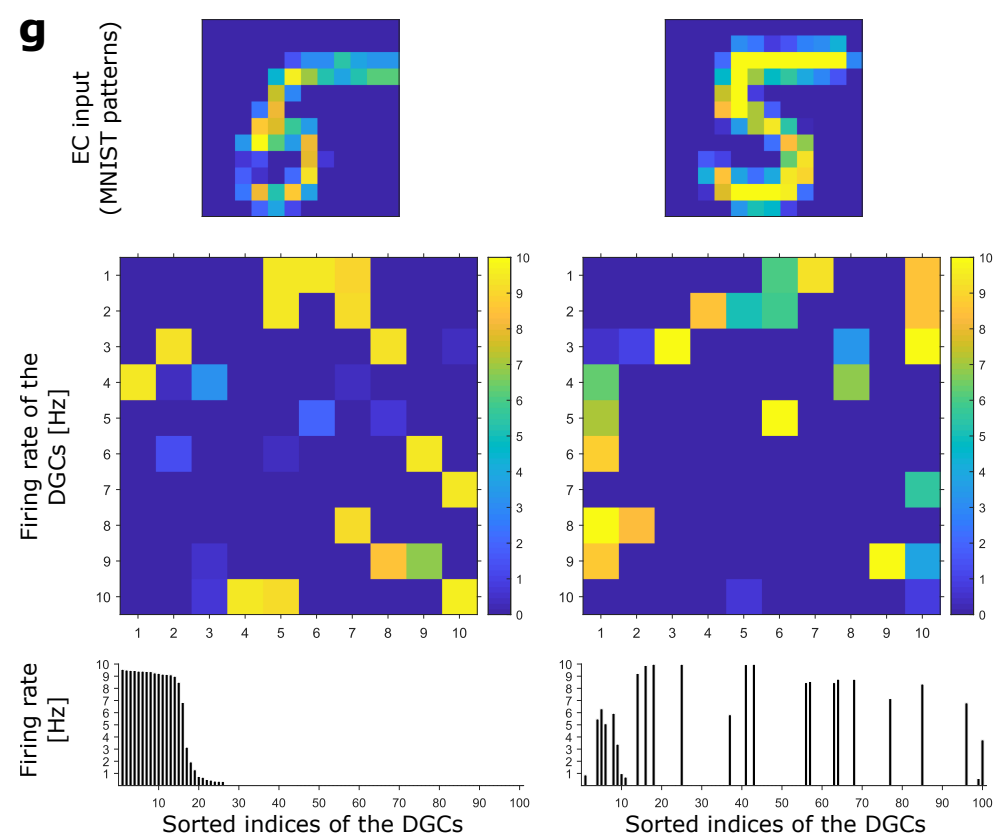
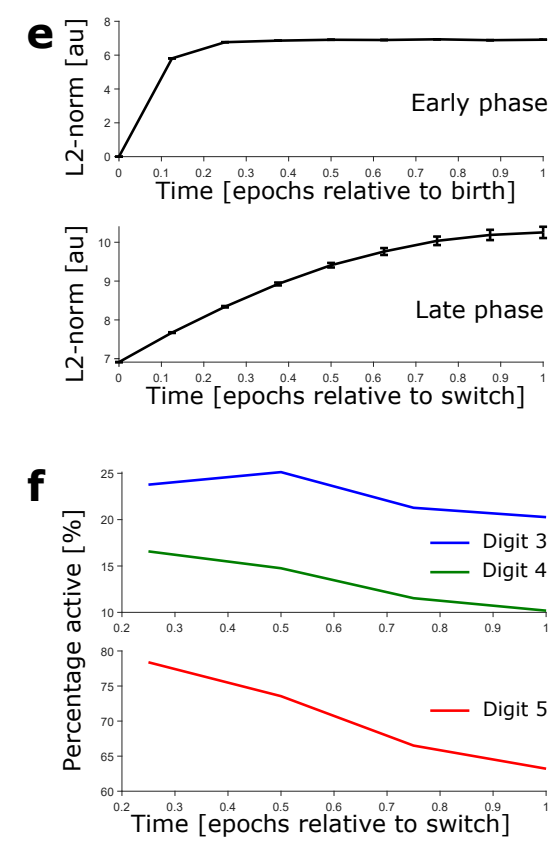
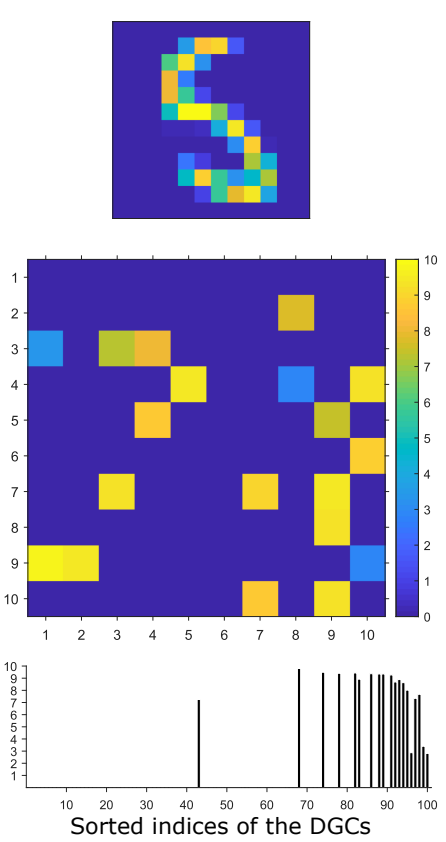

Figure 2: Newborn DGCs become selective for novel patterns during maturation. 
Figure 2: Newborn DGCs become selective for novel patterns during maturation. (a) Unselective neurons are replaced by newborn DGCs, which learn their feedforward weights while patterns from digits 3, 4, and 5 are presented. At the end of the early phase of maturation, the receptive fields of all newborn DGCs (red frames) show mixed selectivity. (b) At the end of the late phase of maturation, newborn DGCs are selective for patterns from the novel digit 5 , with different writing styles. (c,d) Distribution of the percentage of model DGCs (mean with 10th and 90th percentiles) in each firing rate bin at the end of the early (c) and late (d) phase of maturation. Statistics calculated across MNIST patterns ('3's, '4's, '5's). Percentages are per subpopulation (mature and newborn). Note that neurons with firing rate $<1 \mathrm{~Hz}$ for one pattern may fire at medium or high rate for another pattern. (e) The L2-norm of the feedforward weight vector onto newborn DGCs (mean \pm SEM) increases as a function of maturation indicating growth of synapses and receptive field strength. Horizontal axis: time $=1$ indicates end of early (top) or late phase (bottom). (f) Percentage of newborn DGCs activated (firing rate $>1 \mathrm{~Hz}$ ) by a stimulus averaged over all test patterns as a function of maturation. (g) At the end of the late phase of maturation, three different patterns of digit 5 applied to EC neurons (top) cause different firing rate patterns of the 100 DGCs arranged in a matrix of 10-by-10 cells (middle). DGCs with a receptive field (see Fig. 2b) similar to a presented EC activation pattern respond more strongly than the others. Bottom: Firing rates of the DGCs with indices sorted from highest to lowest firing rate in response to the first pattern. All 3 patterns shown come from the testing set, and are correctly classified using our readout network. 

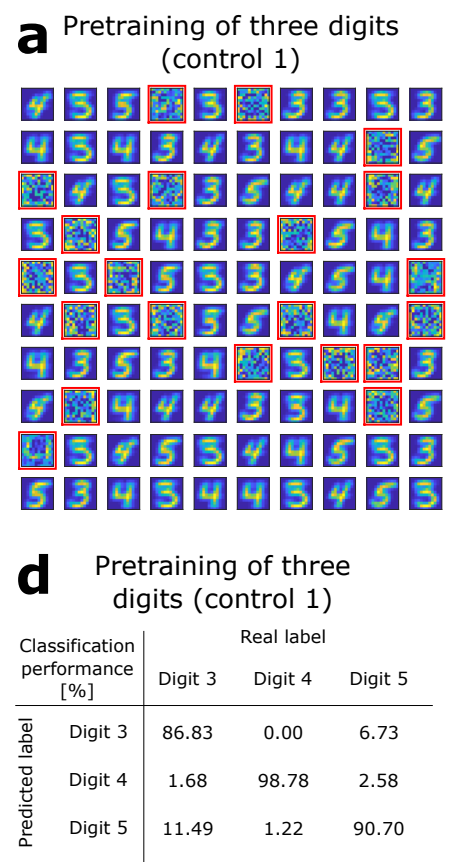

b Only unresponsive DGCs

stay plastic (control 2)

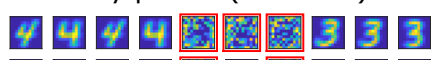

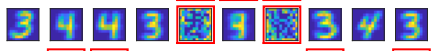

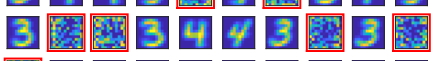

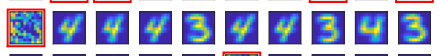

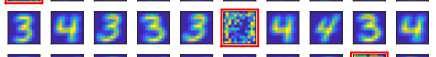

34344443橉

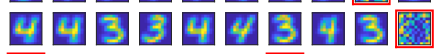

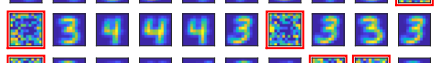

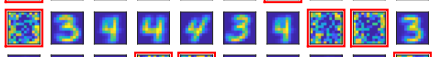

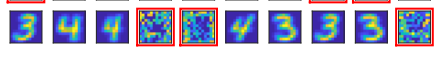

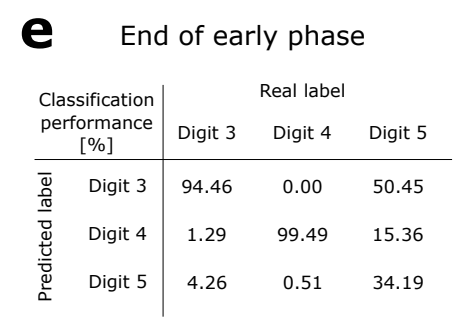

C All DGCs stay plastic (control 3)

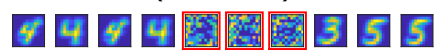

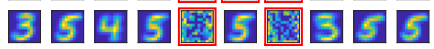

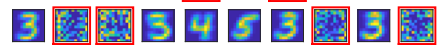

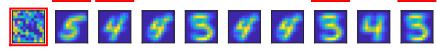
34513圈4654 54349543图

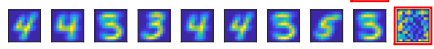

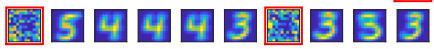

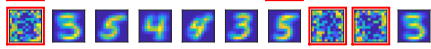

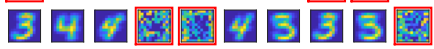

f End of late phase

\begin{tabular}{|c|c|c|c|}
\hline \multirow{2}{*}{$\begin{array}{c}\text { Classification } \\
\text { performance } \\
{[\%]}\end{array}$} & \multicolumn{3}{|c|}{ Real label } \\
\hline & Digit 3 & Digit 4 & Digit 5 \\
\hline Digit 3 & 90.50 & 0.20 & 3.14 \\
\hline Digit 4 & 0.89 & 98.17 & 1.68 \\
\hline Digit 5 & 8.61 & 1.63 & 95.18 \\
\hline
\end{tabular}

Figure 3: The GABA-switch guides learning of novel representations. (a) Pretraining on digits 3,4 and 5 simultaneously without neurogenesis (control 1). Patterns from digits 3,4 and 5 are presented to the network while all DGCs learn their feedforward weights. After pretraining, 79 DGCs have receptive fields corresponding to the three learned digits, while 21 remain unselective (as in Fig. 17). (b) Sequential training without neurogenesis (control 2). After pretraining as in Fig. 1 , the unresponsive neurons stay plastic, but they fail to become selective for digit 5 when patterns from digits 3, 4, and 5 are presented in random order. (c) Sequential training without neurogenesis but all DGCs stay plastic (control 3). Some of the DGCs previously responding to patterns from digits 3 or 4 become selective for digit 5. (d-f) Confusion matrices. Classification performance in percent (using a linear classifier as readout network) for control 1 (d) and for the standard model at the end of the early (e) and late (f) phase; cf. Fig. 2a,b. 

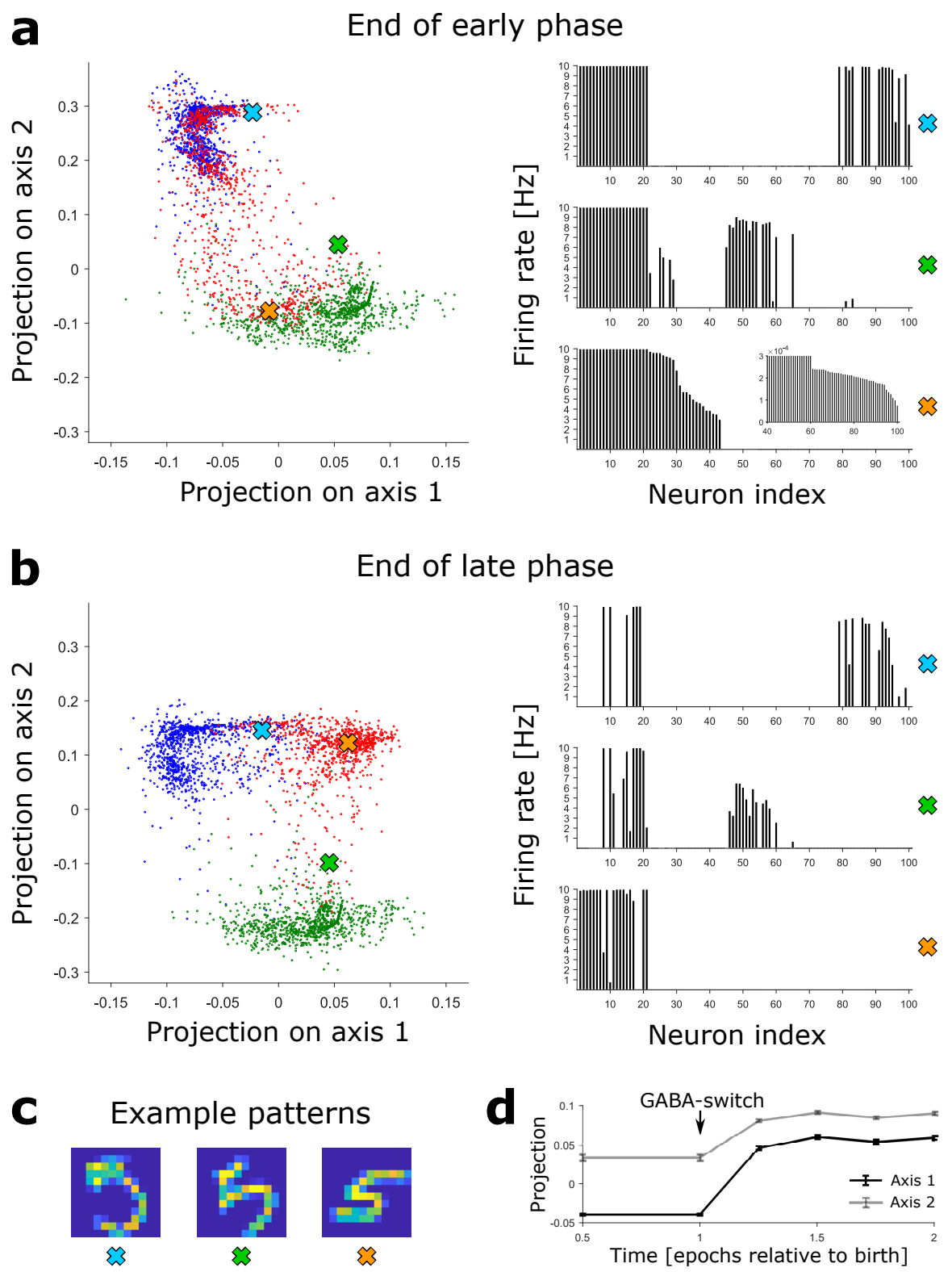

Figure 4: Novel patterns expand the representation into a previously empty subspace. (a) Left: The DGC activity responses at the end of the early phase of maturation of newborn DGCs are projected on discriminatory axes. Each point corresponds to the representation of one input pattern. Color indicates digit 3 (blue), 4 (green), and 5 (red). Right: Firing rate profiles of three example patterns (highlighted by crosses on the left) are sorted from high to low for the pattern represented by the orange cross (inset: zoom of firing rates of DGCs with low activity). (b) Same as a, but at the end of the late phase of maturation of newborn DGCs. Note that the red dots around the orange cross have moved into a different subspace. (c) Example patterns of digit 5 corresponding to the symbols in $\mathbf{a}$ and $\mathbf{b}$. All three are accurately classified by our readout network. (d) Evolution of the mean $( \pm \mathrm{SEM})$ of the projection of the activity upon presentation of all test patterns of digit 5 . 

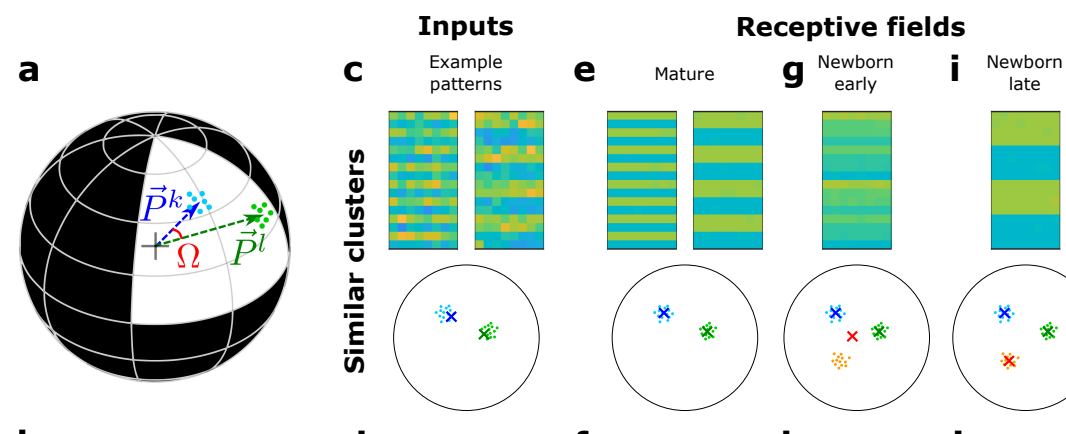

\section{Centers of mass}

k
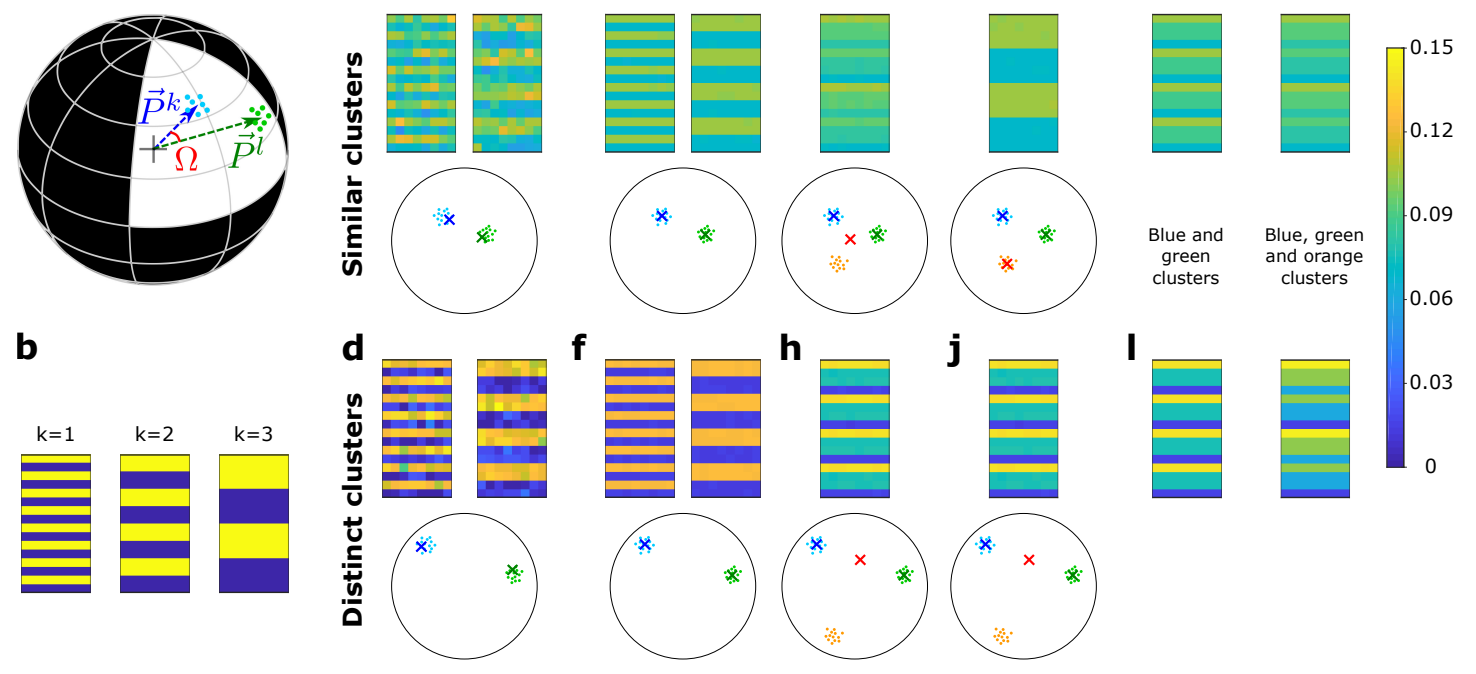

I
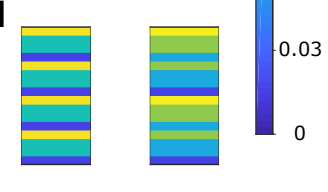

Figure 5: A newborn DGC becomes selective for similar but not distinct novel stimuli. 
Figure 5: A newborn DGC becomes selective for similar but not distinct novel stimuli. (a) Center of mass of clusters $k$ and $l$ of an artificial data set $\left(\vec{P}_{k}\right.$ and $\vec{P}_{l}$, respectively, separated by angle $\Omega$ ) are represented by arrows that point to the surface of a hypersphere. Dots represent individual patterns. (b) Center of mass of three clusters of the artificial data set, visualized as 16x8 pixel patterns. The two-dimensional arrangements and colors are for visualization only. (c,d) Example input patterns (activity of 16x8 input neurons) from clusters 1 and 2 for similar clusters $(\mathbf{c}, s=0.8)$, and distinct clusters $(\mathbf{d}, s=0.2)$. Below: dots correspond to patterns, crosses indicate the input patterns shown (schematic). $(\mathbf{e}, \mathbf{f})$ After pretraining with patterns from two clusters, the receptive fields (set of synaptic weights onto neurons 1 and 2) exhibit the center of mass of each cluster of input patterns (blue and green crosses). (g,h) Novel stimuli from cluster 3 (orange dots) are added. If the clusters are similar, the receptive field of the newborn DGC (red cross) moves towards the center of mass of the three clusters during its early phase of maturation $(\mathrm{g})$, and if the clusters are distinct towards the center of mass of the two pretrained clusters $(\mathbf{h})$. (i,j) Receptive field after the late phase of maturation for the case of similar (i) or distinct (j) clusters. (k,l) For comparison, the center of mass of all patterns of the blue and green clusters (left column) and of the blue, green and orange clusters (right column) for the case of similar (k) or distinct (1) clusters. Color scale: input firing rate $\vec{x}$ or weight $\vec{w}_{i}$ normalized to $\left\|\vec{w}_{i}\right\|=1=\|\vec{x}\|$. 

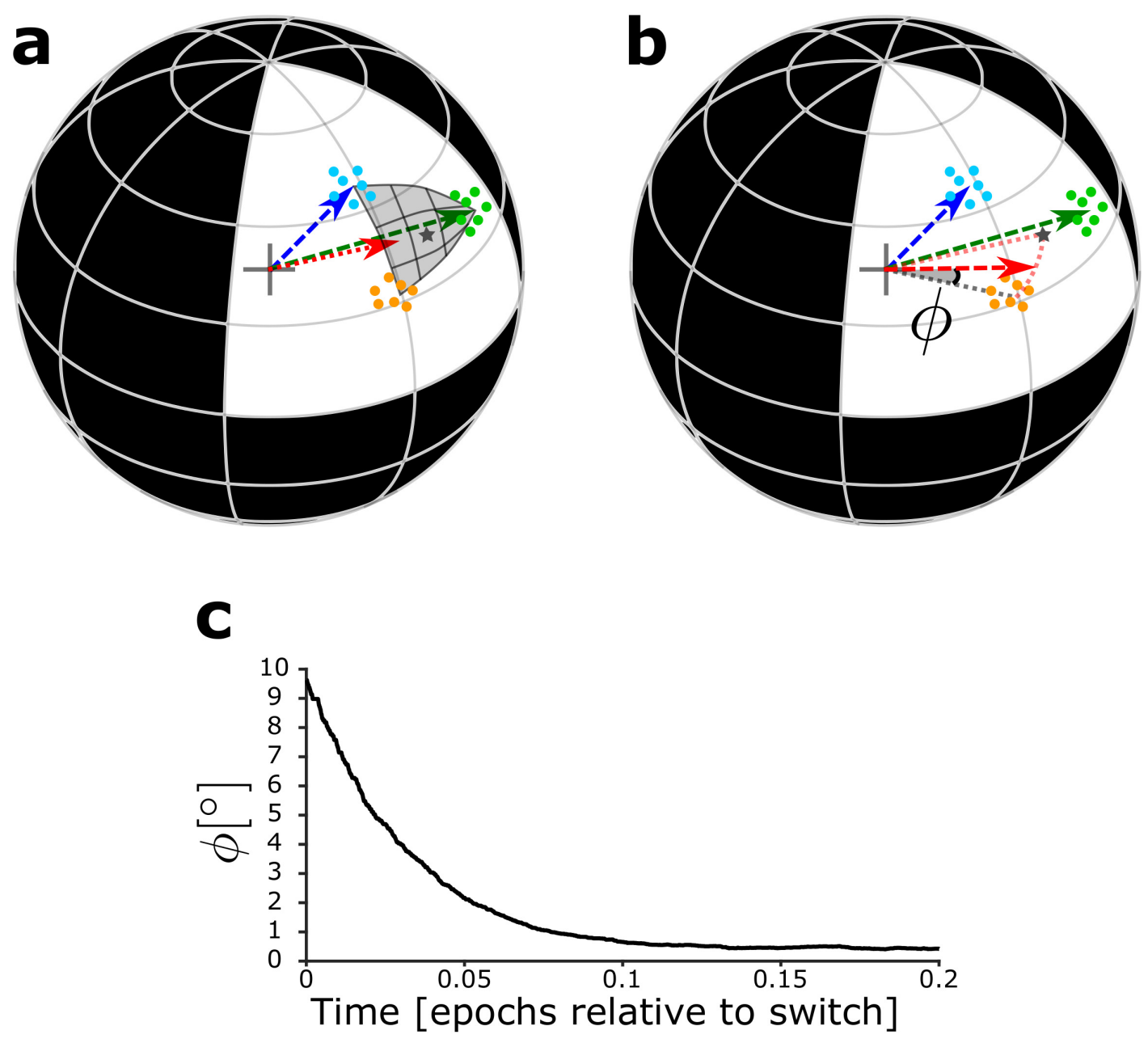

Figure 6: Maturation dynamics for similar patterns. (a) Schematics of the unit hypersphere with three clusters of patterns (colored dots) and three scaled feedforward weight vectors (colored arrows). After pretraining, the blue and green weight vectors point to the center of mass of the corresponding clusters. Patterns from the novel cluster (orange points) are presented only later to the network. During the early phase of maturation, the newborn DGC grows its vector of feedforward weights (red arrow) in the direction of the subspace of patterns which indirectly activate the newborn cell (dark grey star: center of mass of the presented patterns, located below the part of the sphere surface highlighted in grey). (b) During the late phase of maturation, the red vector turns towards the novel cluster. Angle $\phi$ between the center of mass of the novel cluster and the feedforward weight vector onto the newborn cell. (c) The angle $\phi$ decreases in the late phase of maturation of the newborn DGC if the novel cluster is similar to the previously stored clusters. Its final average value of $\phi \approx 0.4^{\circ}$ is caused by the jitter of the weight vector around the center of mass of the novel cluster. 


\section{Table 1: Parameters for the simulations}

\begin{tabular}{c|cc|cc} 
& \multicolumn{2}{|c|}{ Biologically-plausible network } & \multicolumn{2}{c}{ Simplified network } \\
\hline \multirow{2}{*}{ Network } & $N_{E C}=144$ & $N_{D G C}=100$ & $N_{E C}=128$ & $N_{D G C}=3$ \\
& $N_{I}=25$ & & \\
& $w_{I E}=1$ & $w_{E I}=-\frac{1}{p_{E I} * N_{I}}$ & $w_{\text {rec }}=-1.2$ & \\
Connectivity & $p_{I E}=0.9$ & $p_{E I}=0.9$ & \\
Dynamics & $\tau_{m}=20 \mathrm{~ms}$ & $\tau_{\text {inh }}=2 \mathrm{~ms}$ & $\tau_{m}=20 \mathrm{~ms}$ & \\
& $L=0.5$ & $p^{*}=0.1$ & & \\
Plasticity & $\alpha_{0}=0.05$ & $\beta=1$ & $\alpha_{0}=0.03$ & $\beta=1$ \\
& $\gamma_{0}=10$ & $\theta=0.15$ & $\gamma_{0}=1.65$ & $\theta=0.15$ \\
& $\nu_{0}=0.2$ & $\gamma=9.85$ & $\gamma=1.5$ & \\
Numerical simulations & $\Delta t=0.1 \mathrm{~ms}$ & $\eta=0.01$ & $\Delta t=1 \mathrm{~ms}$ & $\eta=0.01$ \\
& $\eta_{b}=0.01$ & & &
\end{tabular}

